\title{
Highly excited and exotic meson spectroscopy from lattice QCD
}

\section{Christopher Thomas, Jefferson Lab}

\section{thomasc@jlab.org}

\section{Confinement IX, Madrid, August 2010}

With Jo Dudek, Robert Edwards, Mike Peardon, David Richards and the Hadron Spectrum Collaboration 


\section{Outline}

- Introduction and motivation

- Method outline - excited spectra from LQCD

- Results - isovector and kaon spectra

- Summary and outlook 


\section{Motivation}

Renaissance in excited charmonium spectroscopy

BABAR, Belle, BES, CLEO-c, ...

Upcoming experimental efforts (in charmonium and light meson sector)

GlueX (JLab), BESIII, PANDA, ... 


\section{Motivation}

Renaissance in excited charmonium spectroscopy

BABAR, Belle, BES, CLEO-c, ...

Upcoming experimental efforts (in charmonium and light meson sector) GlueX (JLab), BESIII, PANDA, ...

$$
\begin{array}{r}
\operatorname{Exotics}\left(\mathrm{JPC}^{\mathrm{PC}}=\mathbf{1}^{-+}, \mathbf{2}^{+-}, \ldots\right) \text { ? } \quad-\text { can't just be a } q \bar{q} \text { pair } \\
\text { e.g. hybrids, multi-mesons }
\end{array}
$$




\section{Motivation}

Renaissance in excited charmonium spectroscopy

BABAR, Belle, BES, CLEO-c, ...

Upcoming experimental efforts (in charmonium and light meson sector) GlueX (JLab), BESIII, PANDA, ...

Exotics $\left(\mathrm{JPC}^{\mathrm{PC}}=\mathbf{1}^{-+}, \mathbf{2}^{+-}, \ldots\right)$ ? - can't just be a $q \bar{q}$ pair e.g. hybrids, multi-mesons

Two spin-half fermions: $\quad{ }^{2 S+1} L_{J}$

Parity:

$$
P=(-1)^{(L+1)}
$$

Charge Conj Sym: $C=(-1)^{(L+S)}$

$$
\mathrm{JPC}^{\mathrm{PC}} 0^{-+}, \mathrm{O}^{++}, 1^{--}, 1^{++}, 1^{+-}, 2^{--}, 2^{++}, 2^{-+}, \ldots
$$




\section{Motivation}

Renaissance in excited charmonium spectroscopy

BABAR, Belle, BES, CLEO-c, ...

Upcoming experimental efforts (in charmonium and light meson sector) GlueX (JLab), BESIII, PANDA, ...

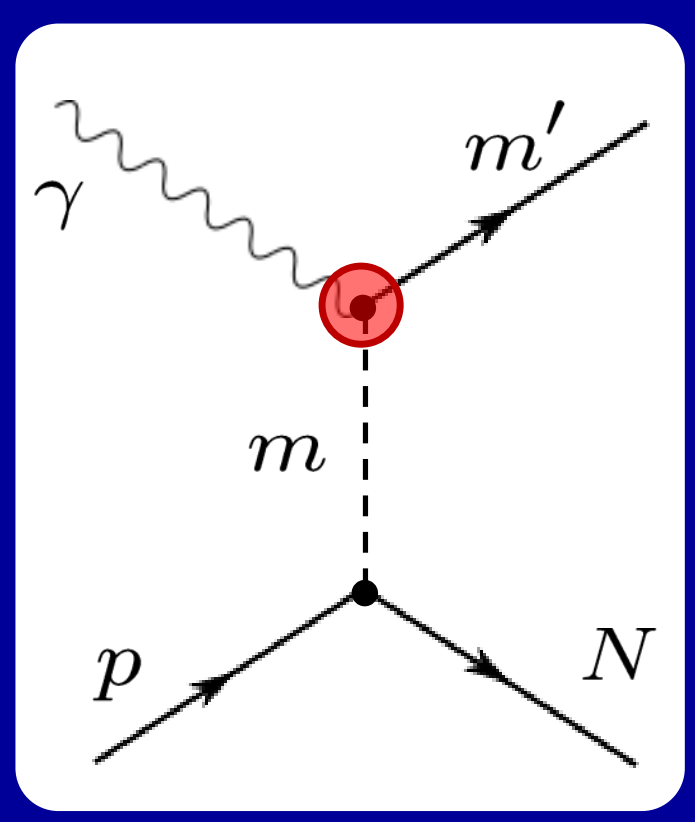

$\operatorname{Exotics}\left(\mathrm{JPC}^{\mathrm{PC}}=\mathbf{1}^{-+}, \mathbf{2}^{+-}, \ldots\right)$ ? - can't just be a $q \bar{q}$ pair e.g. hybrids, multi-mesons

Photoproduction at GlueX (JLab 12 GeV upgrade) 


\section{Motivation}

Renaissance in excited charmonium spectroscopy

BABAR, Belle, BES, CLEO-c, ...

Upcoming experimental efforts (in charmonium and light meson sector) GlueX (JLab), BESIII, PANDA, ...

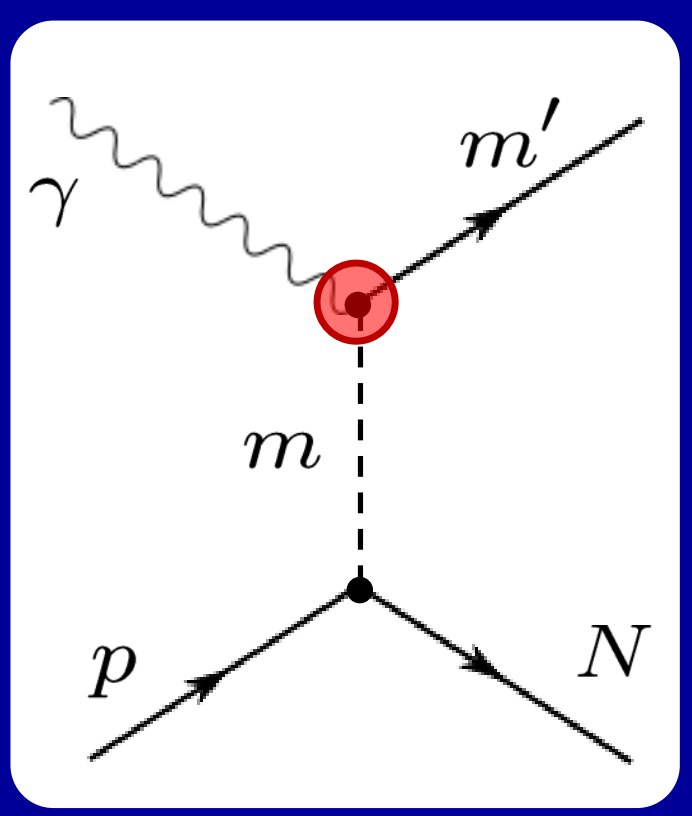

Exotics $\left(\mathrm{JPC}^{\mathrm{PC}}=1^{-+}, 2^{+-}, \ldots\right)$ ? - can't just be a $q \bar{q}$ pair e.g. hybrids, multi-mesons

Photoproduction at GlueX (JLab 12 GeV upgrade)

Use Lattice QCD to extract excited spectrum...

... and photocouplings (tested in charmonium) 


\section{Spectroscopy on the lattice}

Calculate energies and matrix elements ("overlaps", Z's) from correlation functions of meson interpolating fields

$$
C_{i j}(t)=<0\left|O_{i}(t) O_{j}(0)\right| 0>
$$




\section{Spectroscopy on the lattice}

Calculate energies and matrix elements ("overlaps", Z's) from correlation functions of meson interpolating fields

$$
C_{i j}(t)=<0\left|O_{i}(t) O_{j}(0)\right| 0>
$$

Construct operators which only overlap on to one spin in the continuum limit

$$
O(t)=\sum_{\vec{x}} e^{i \vec{p} \cdot \vec{x}} \bar{\psi}(x) \Gamma_{i} \overleftrightarrow{D}_{j} \overleftrightarrow{D}_{k} \ldots \psi(x)
$$




\section{Spectroscopy on the lattice}

Calculate energies and matrix elements ("overlaps", Z's) from correlation functions of meson interpolating fields

$$
C_{i j}(t)=<0\left|O_{i}(t) O_{j}(0)\right| 0>
$$

Construct operators which only overlap on to one spin in the continuum limit

$$
O(t)=\sum_{\vec{x}} e^{i \vec{p} \cdot \vec{x}} \bar{\psi}(x) \Gamma_{i} \overleftrightarrow{D}_{j} \overleftrightarrow{D}_{k} \ldots \psi(x)
$$

'Distillation' technology for constructing on lattice PR D80 054506 (2009)

$(p=0) \quad$ definite JPC

$$
C_{i j}(t)=\sum_{n} \frac{e^{-E_{n} t}}{2 E_{n}}<0\left|O_{i}(0)\right| n><n\left|O_{j}(0)\right| 0>
$$




\section{Variational Method}

Large basis of operators $\rightarrow$ matrix of correlators

$$
C_{i j}(t)=<0\left|O_{i}(t) O_{j}(0)\right| 0>
$$

Generalised eigenvector problem:

$$
C_{i j}(t) v_{j}^{(n)}=\lambda^{(n)}(t) C_{i j}\left(t_{0}\right) v_{j}^{(n)}
$$




\section{Variational Method}

Large basis of operators $\rightarrow$ matrix of correlators

Generalised eigenvector problem:

$$
C_{i j}(t)=<0\left|O_{i}(t) O_{j}(0)\right| 0>
$$

$$
C_{i j}(t) v_{j}^{(n)}=\lambda^{(n)}(t) C_{i j}\left(t_{0}\right) v_{j}^{(n)}
$$

Eigenvalues $\rightarrow$ energies

$$
\lambda^{(n)}(t) \rightarrow e^{-E_{n}\left(t-t_{0}\right)} \quad\left(t>t_{0}\right)
$$




\section{Variational Method}

Large basis of operators $\rightarrow$ matrix of correlators

Generalised eigenvector problem:

$$
C_{i j}(t)=<0\left|O_{i}(t) O_{j}(0)\right| 0>
$$

$$
C_{i j}(t) v_{j}^{(n)}=\lambda^{(n)}(t) C_{i j}\left(t_{0}\right) v_{j}^{(n)}
$$

Eigenvalues $\rightarrow$ energies

$$
\lambda^{(n)}(t) \rightarrow e^{-E_{n}\left(t-t_{0}\right)} \quad\left(t>>t_{0}\right)
$$

Eigenvectors $\rightarrow$ optimal linear combination of operators to overlap on to a state

$$
\Omega^{(n)} \sim \sum_{i} v_{i}^{(n)} O_{i}
$$

$Z^{(n)}$ related to eigenvectors

$$
Z_{i}^{(n)} \equiv<0\left|O_{i}\right| n>
$$




\section{Variational Method}

Large basis of operators $\rightarrow$ matrix of correlators

Generalised eigenvector problem:

$$
C_{i j}(t)=<0\left|O_{i}(t) O_{j}(0)\right| 0>
$$

$$
C_{i j}(t) v_{j}^{(n)}=\lambda^{(n)}(t) C_{i j}\left(t_{0}\right) v_{j}^{(n)}
$$

Eigenvalues $\rightarrow$ energies

$$
\lambda^{(n)}(t) \rightarrow e^{-E_{n}\left(t-t_{0}\right)} \quad\left(t>>t_{0}\right)
$$

Eigenvectors $\rightarrow$ optimal linear combination of operators to overlap on to a state

$$
\Omega^{(n)} \sim \sum_{i} v_{i}^{(n)} O_{i}
$$

$Z^{(n)}$ related to eigenvectors

$$
Z_{i}^{(n)} \equiv<0\left|O_{i}\right| n>
$$

Var. method uses orthog of eigenvectors; don't just rely on separating energies 


\section{Light Meson Spectroscopy}

- Dynamical calculation (unquenched - includes light quark loops)

- Anisotropic - finer in temporal direction $\left(a_{s} / a_{t}=3.5, a_{s} \sim 0.12 \mathrm{fm}\right)$

- Only connected diagrams - isovectors $(\mathrm{I}=1)$ and kaons 


\section{Light Meson Spectroscopy}

- Dynamical calculation (unquenched - includes light quark loops)

- Anisotropic - finer in temporal direction $\left(a_{s} / a_{t}=3.5, a_{s} \sim 0.12 \mathrm{fm}\right)$

- Only connected diagrams - isovectors $(I=1)$ and kaons

- As an example: three degenerate 'light' quarks $\left(\mathrm{N}_{\mathrm{f}}=3, \mathrm{M}_{\pi} \approx 700 \mathrm{MeV}\right)$

- Also $\left(\mathrm{N}_{\mathrm{f}}=2+1\right) \mathrm{M}_{\pi} \approx 520,440,400 \mathrm{MeV}$ 


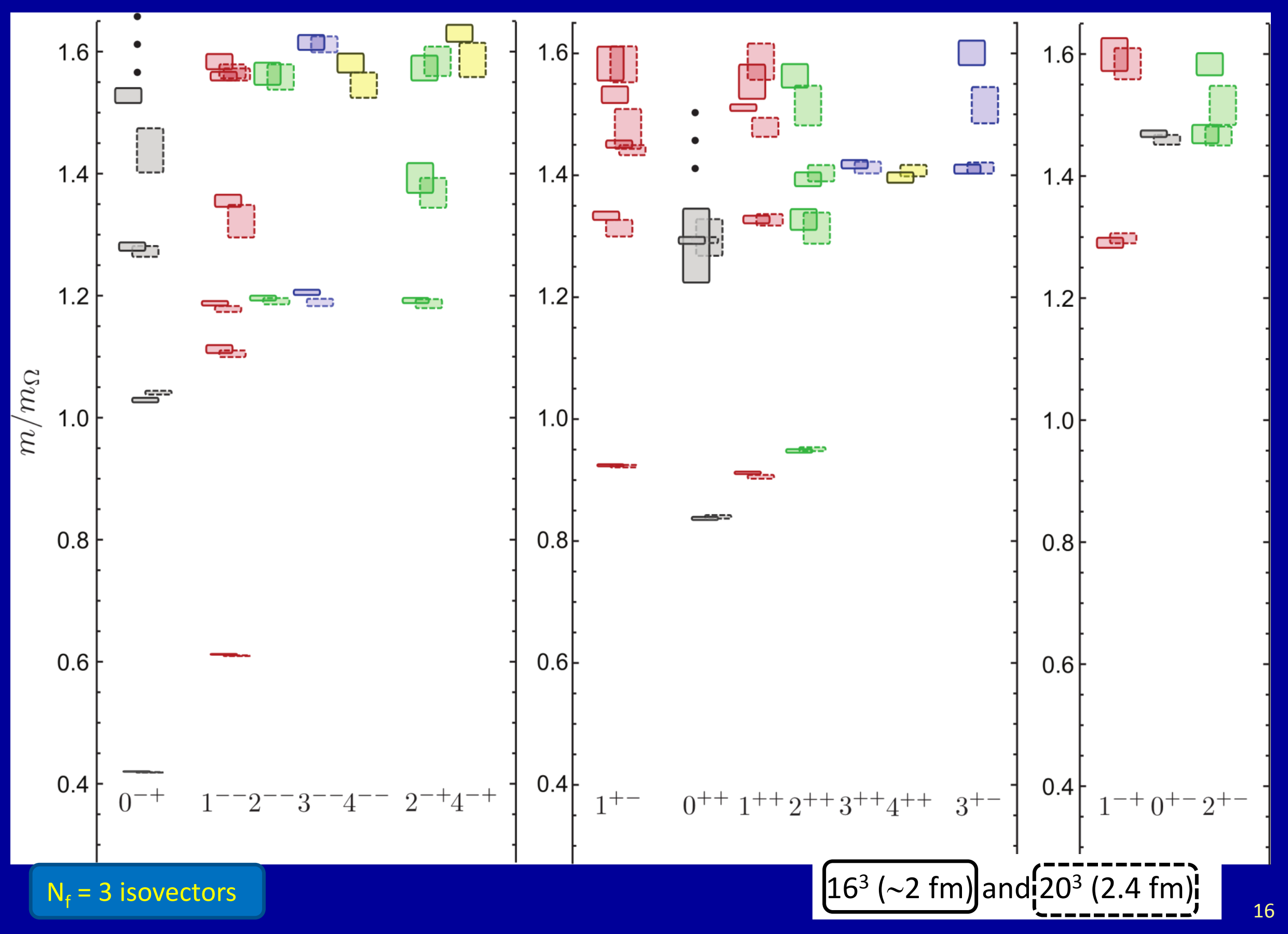




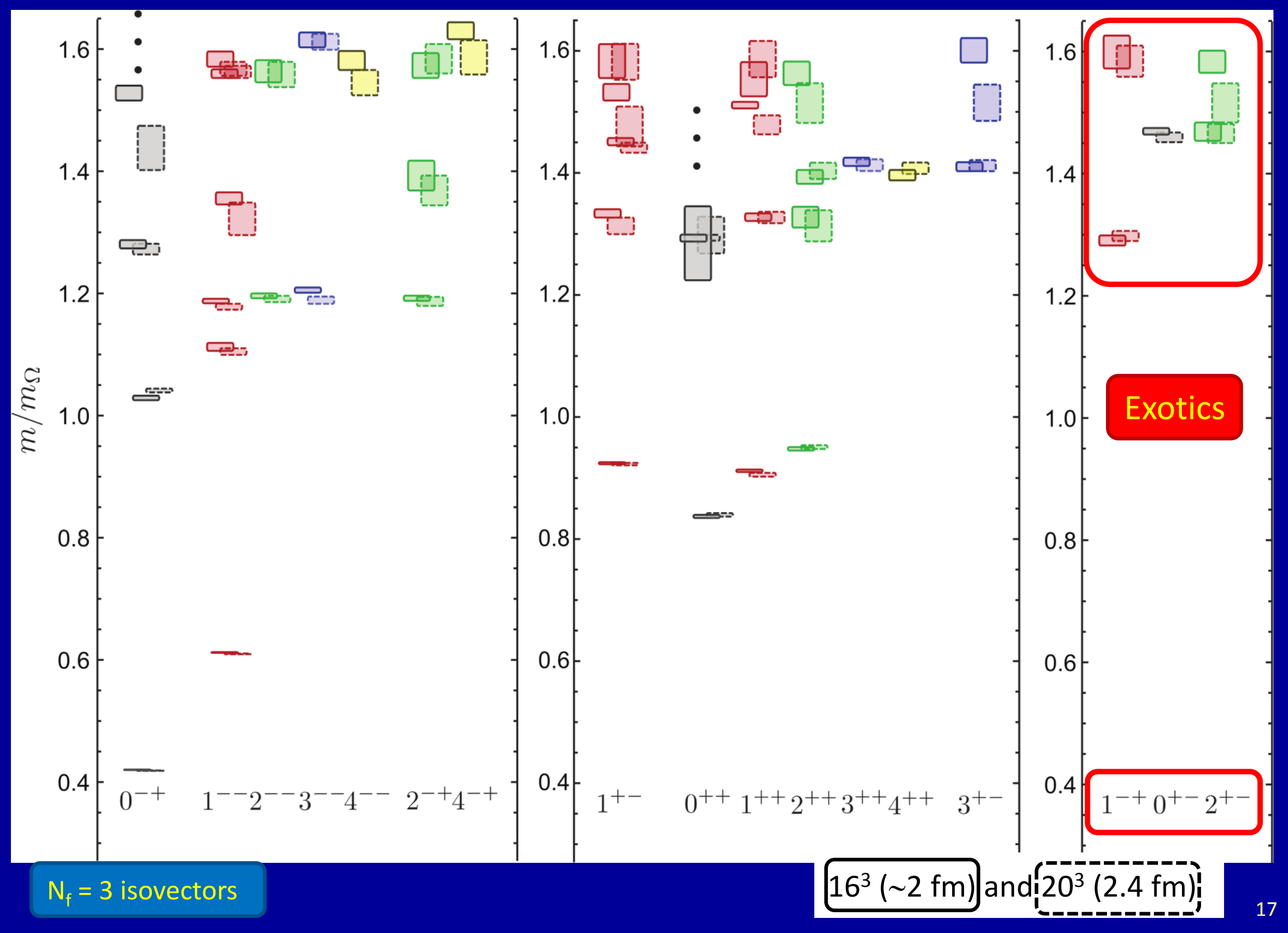




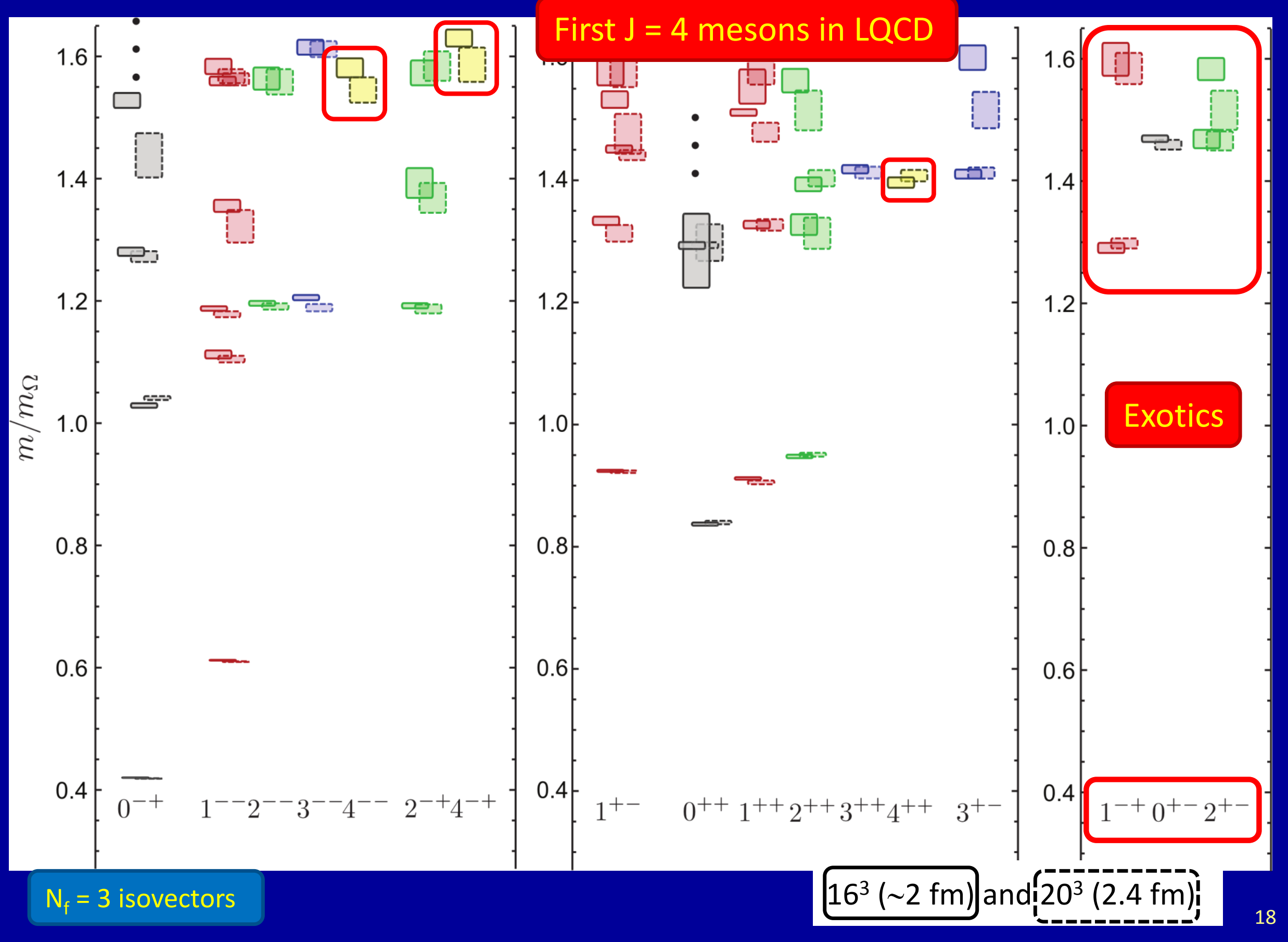




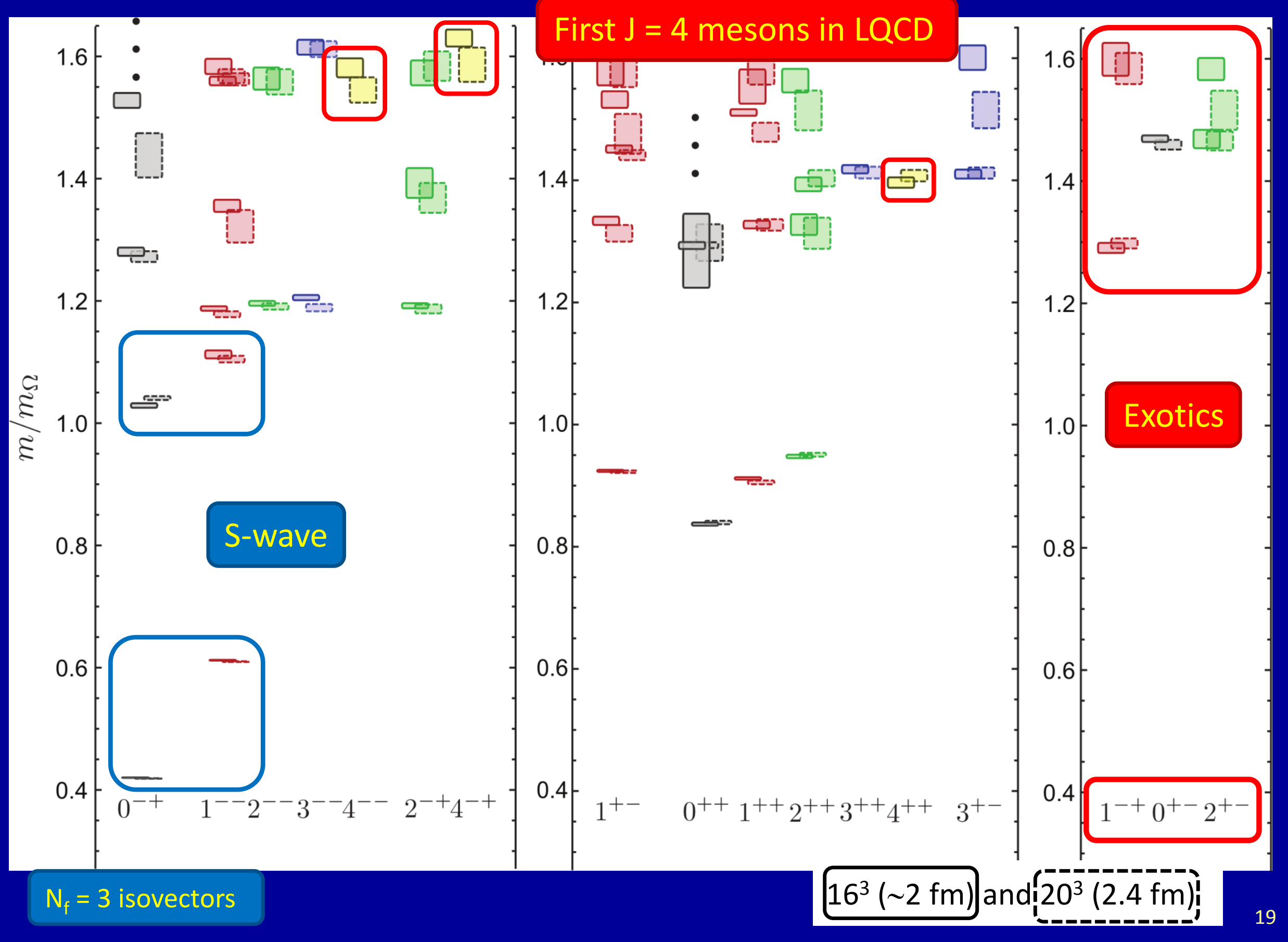




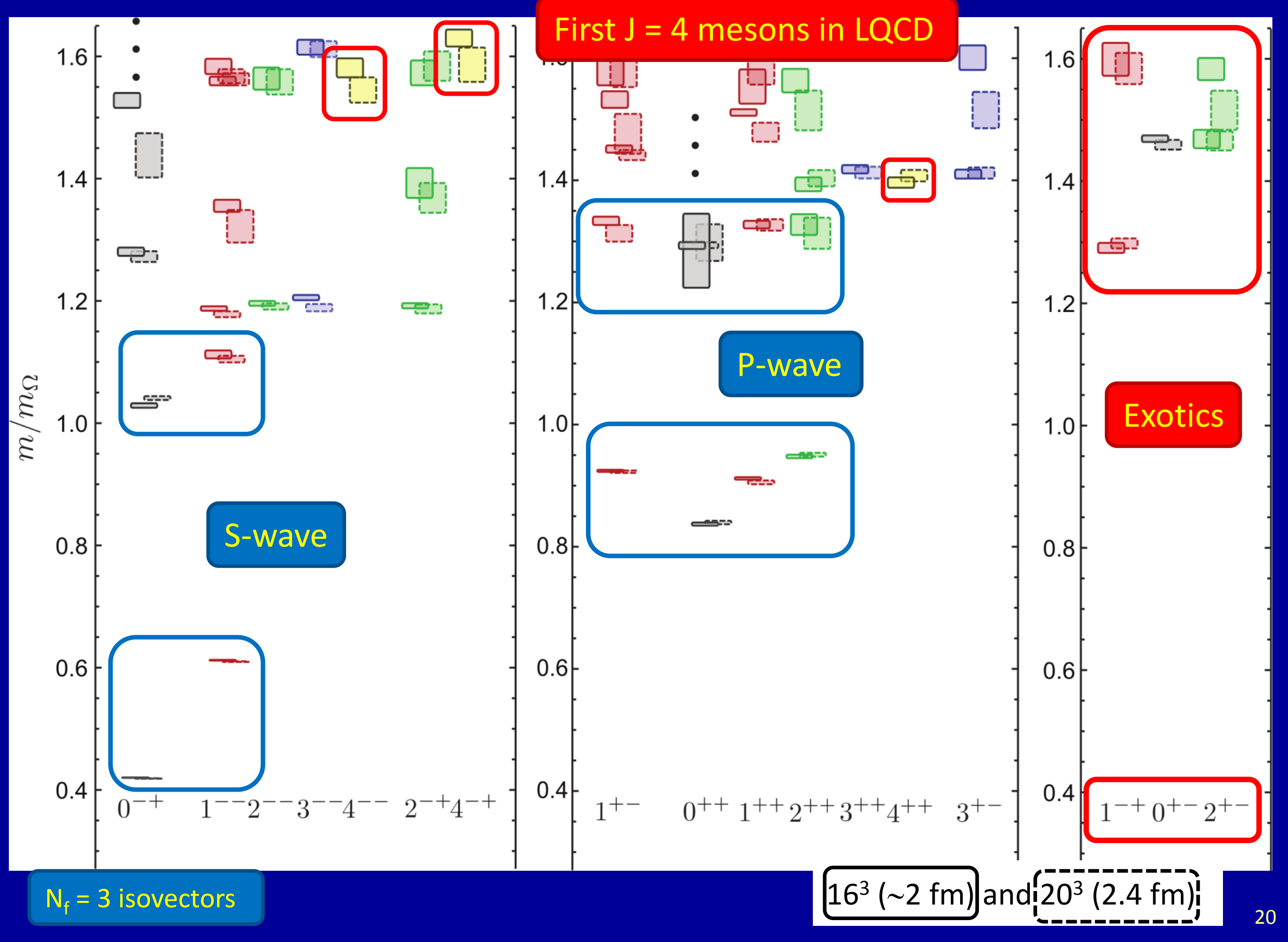




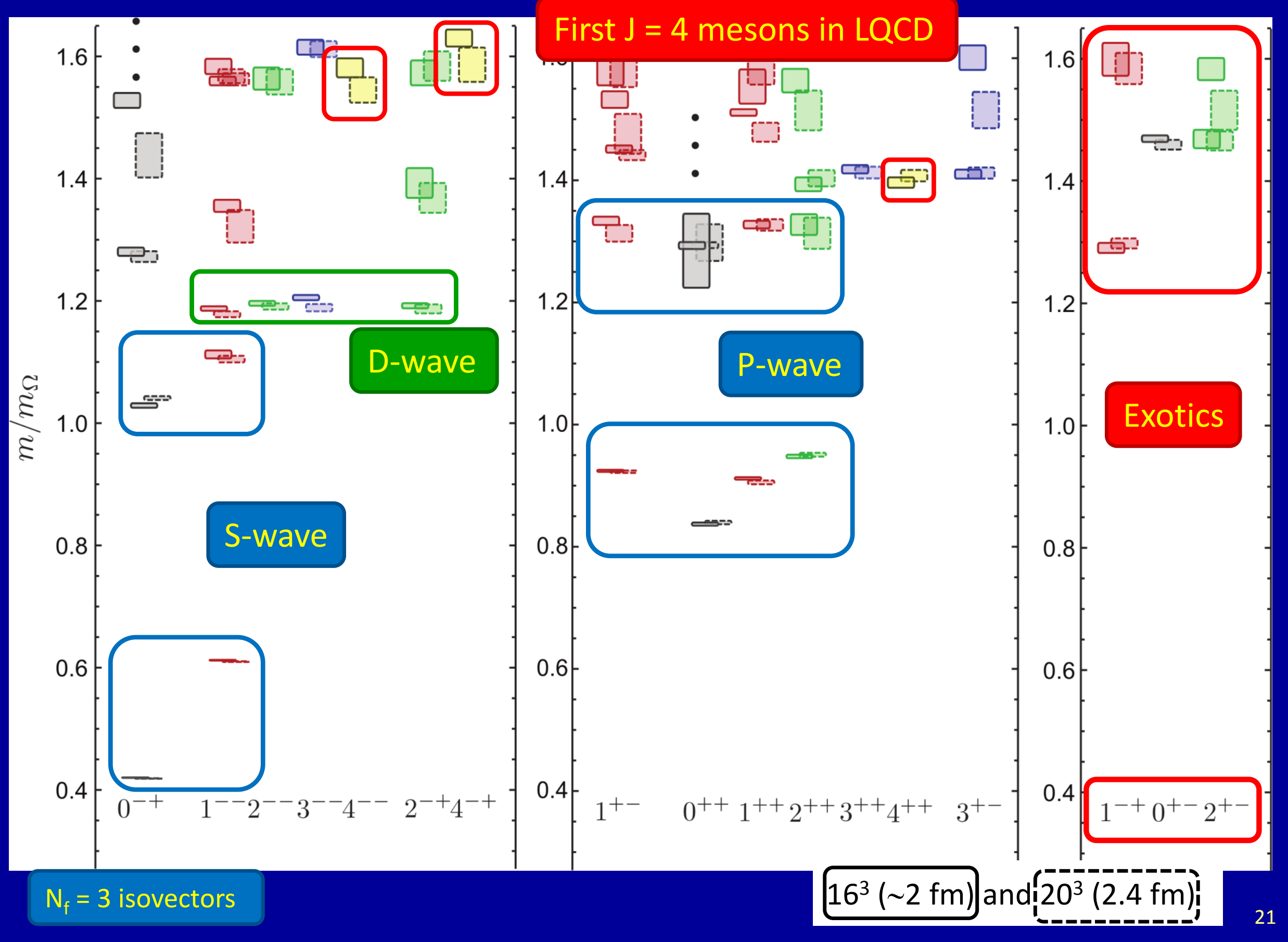




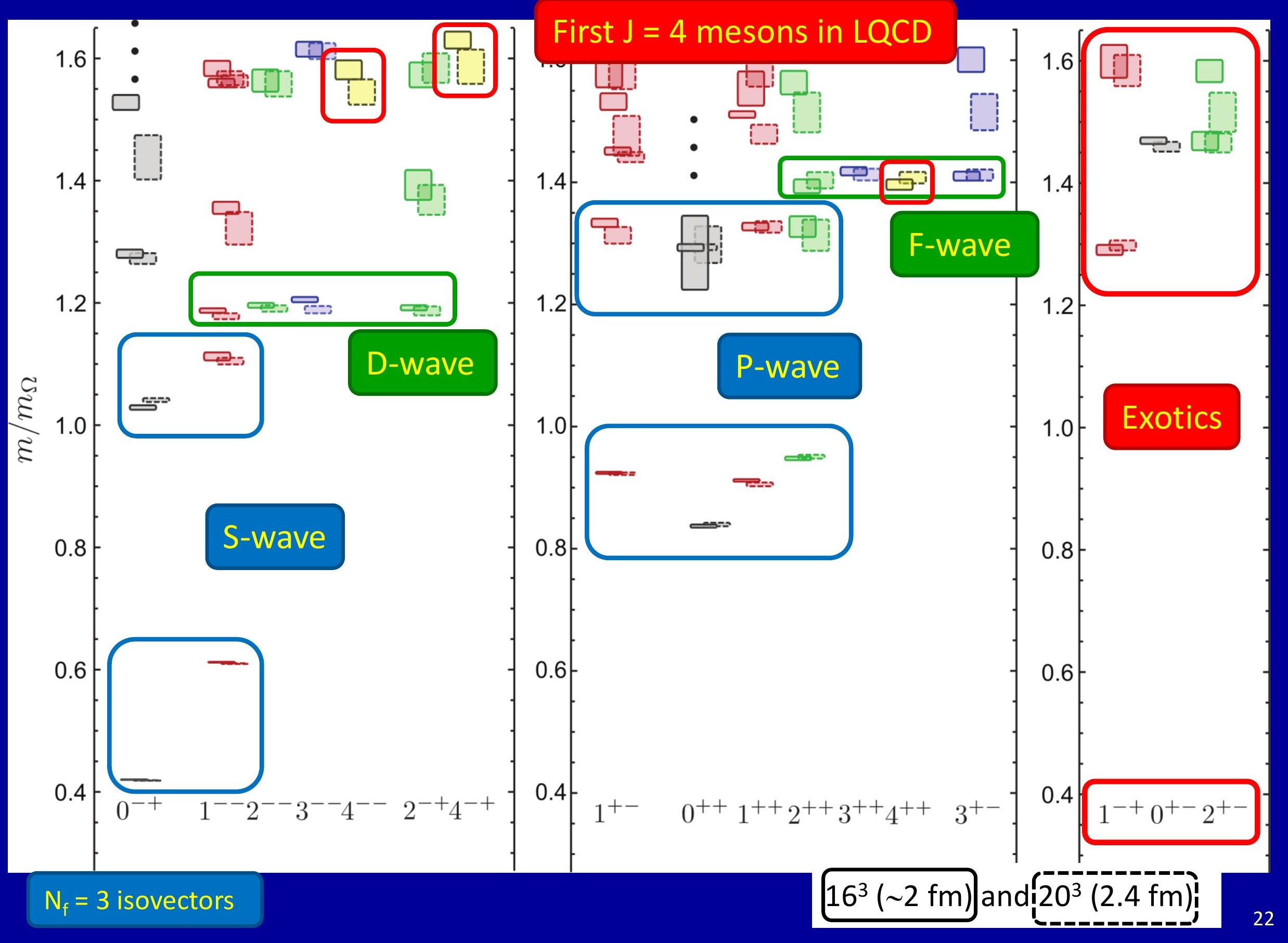




\section{$\mathrm{D}+\mathrm{G}$-waves}

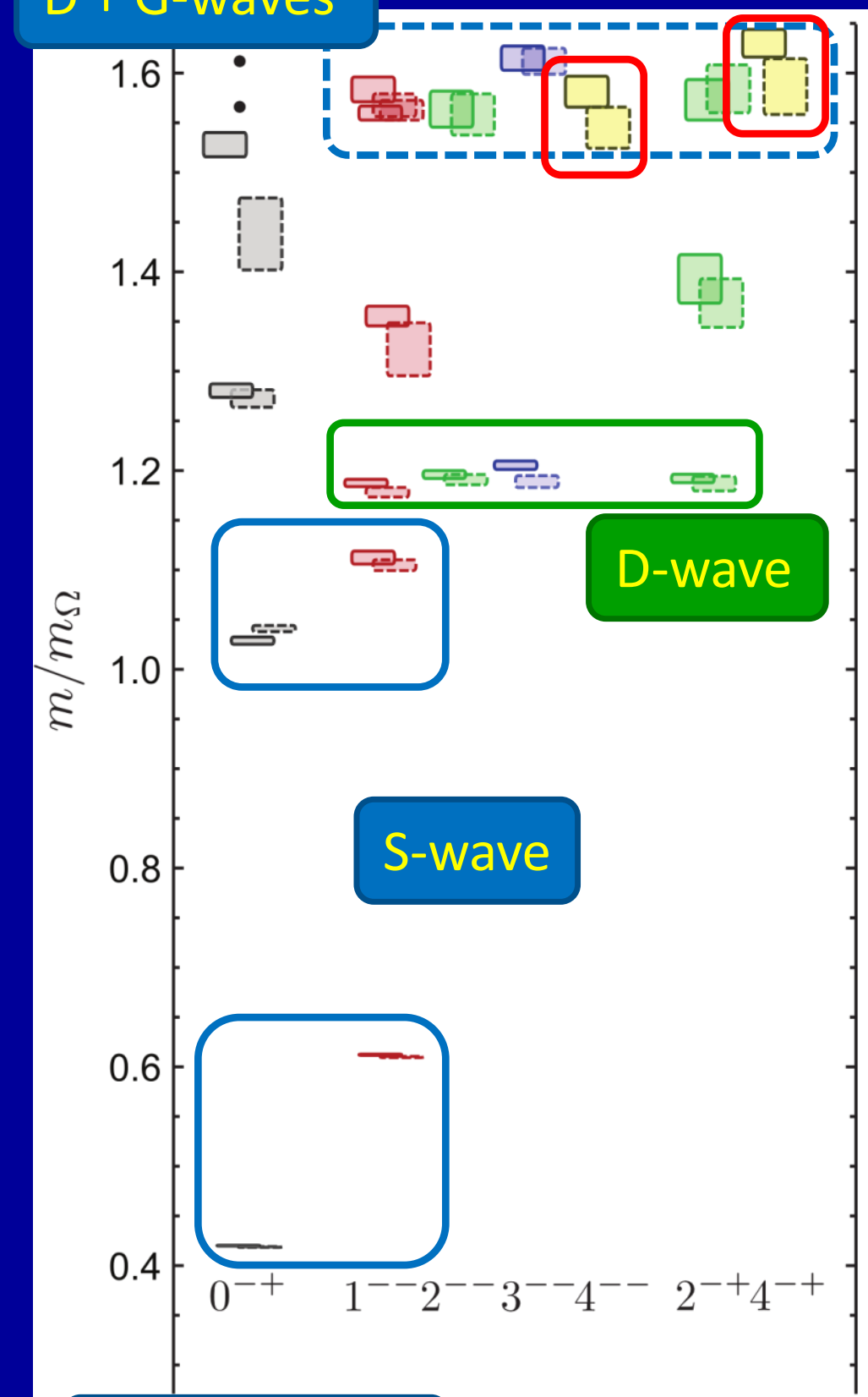

$N_{f}=3$ isovectors

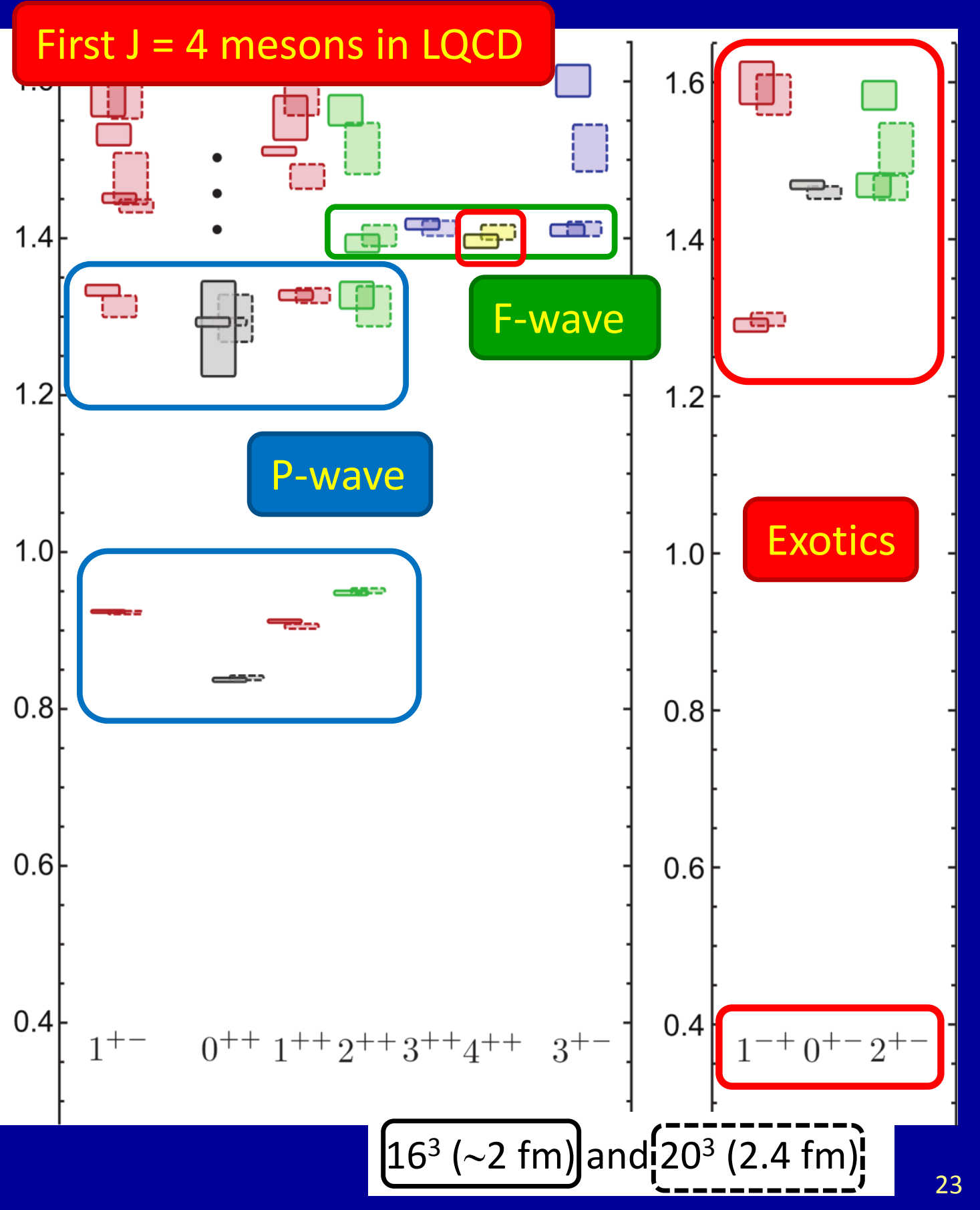




\section{$\mathrm{D}+\mathrm{G}$-waves}

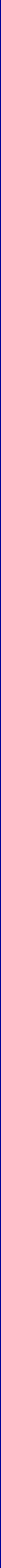




\section{Lower pion masses}

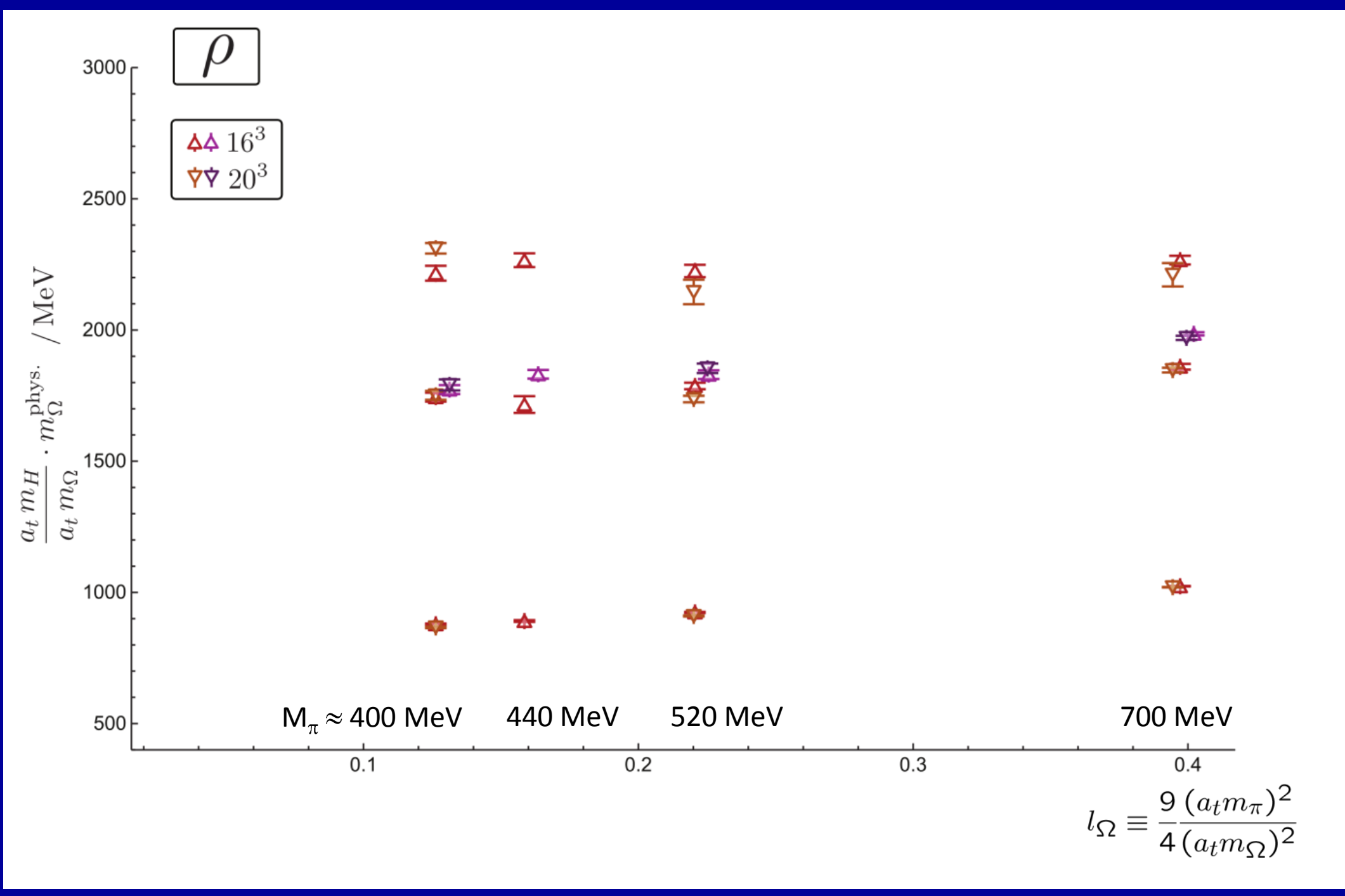




\section{Lower pion masses}

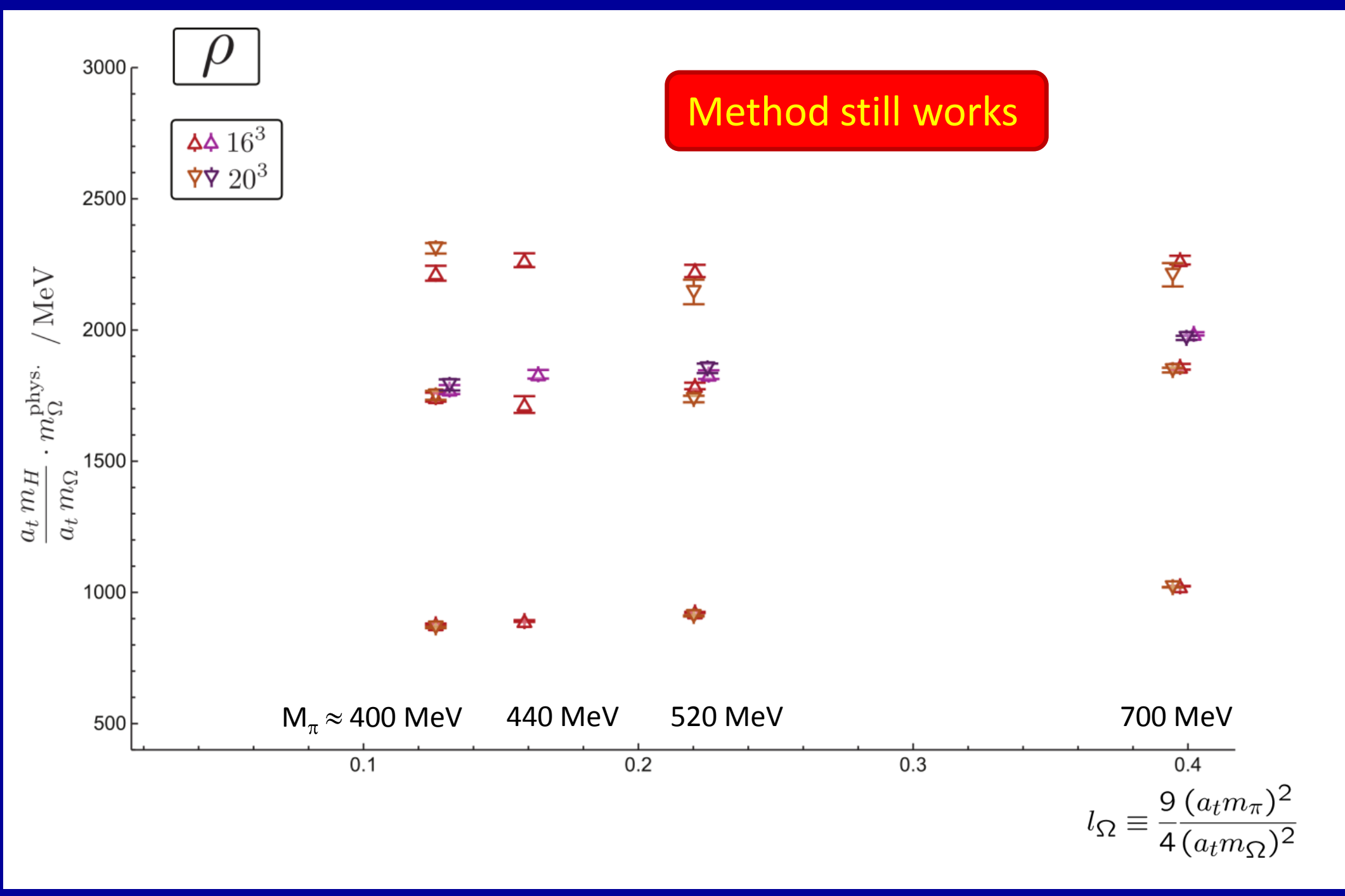



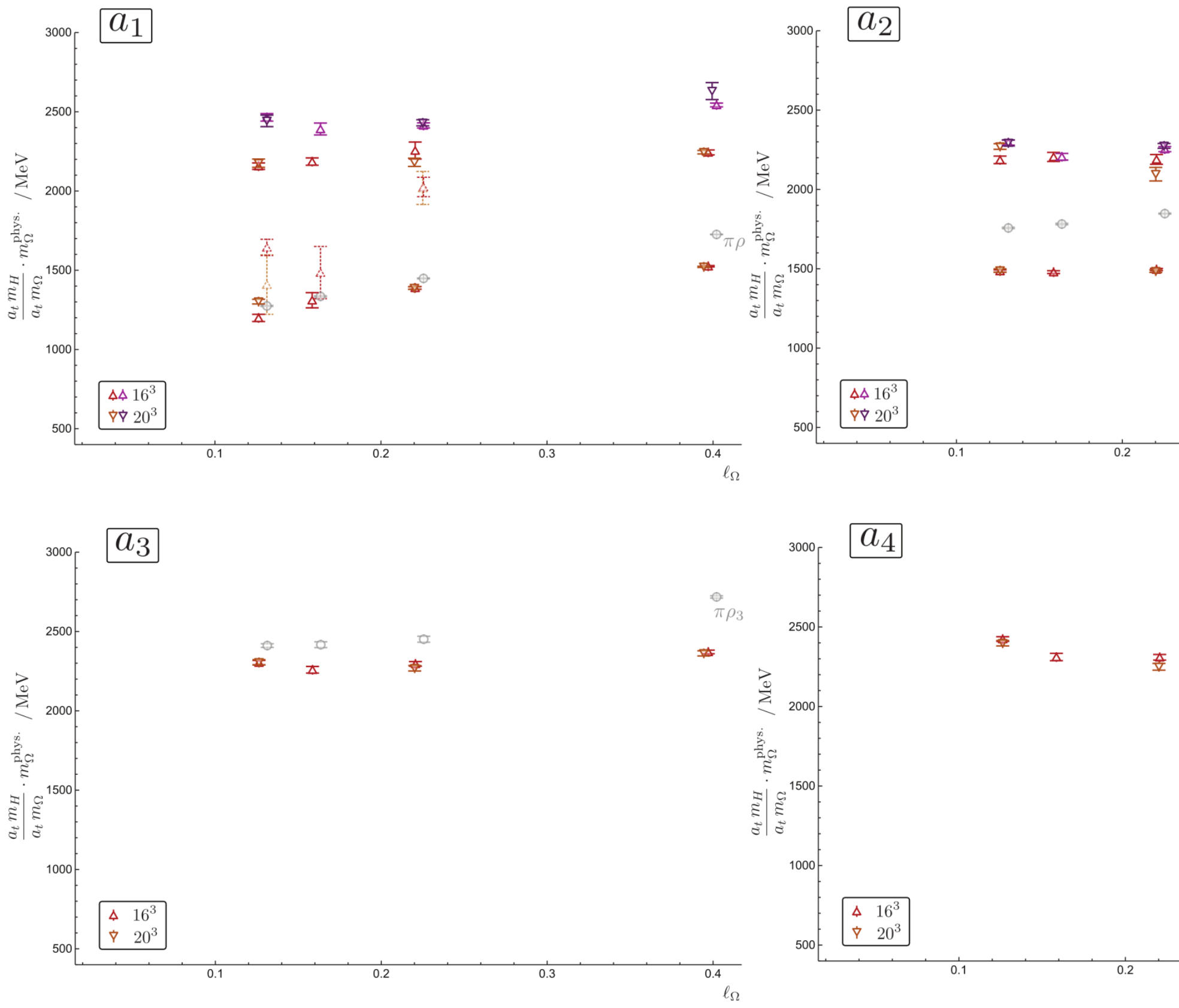


\section{Exotics summary}

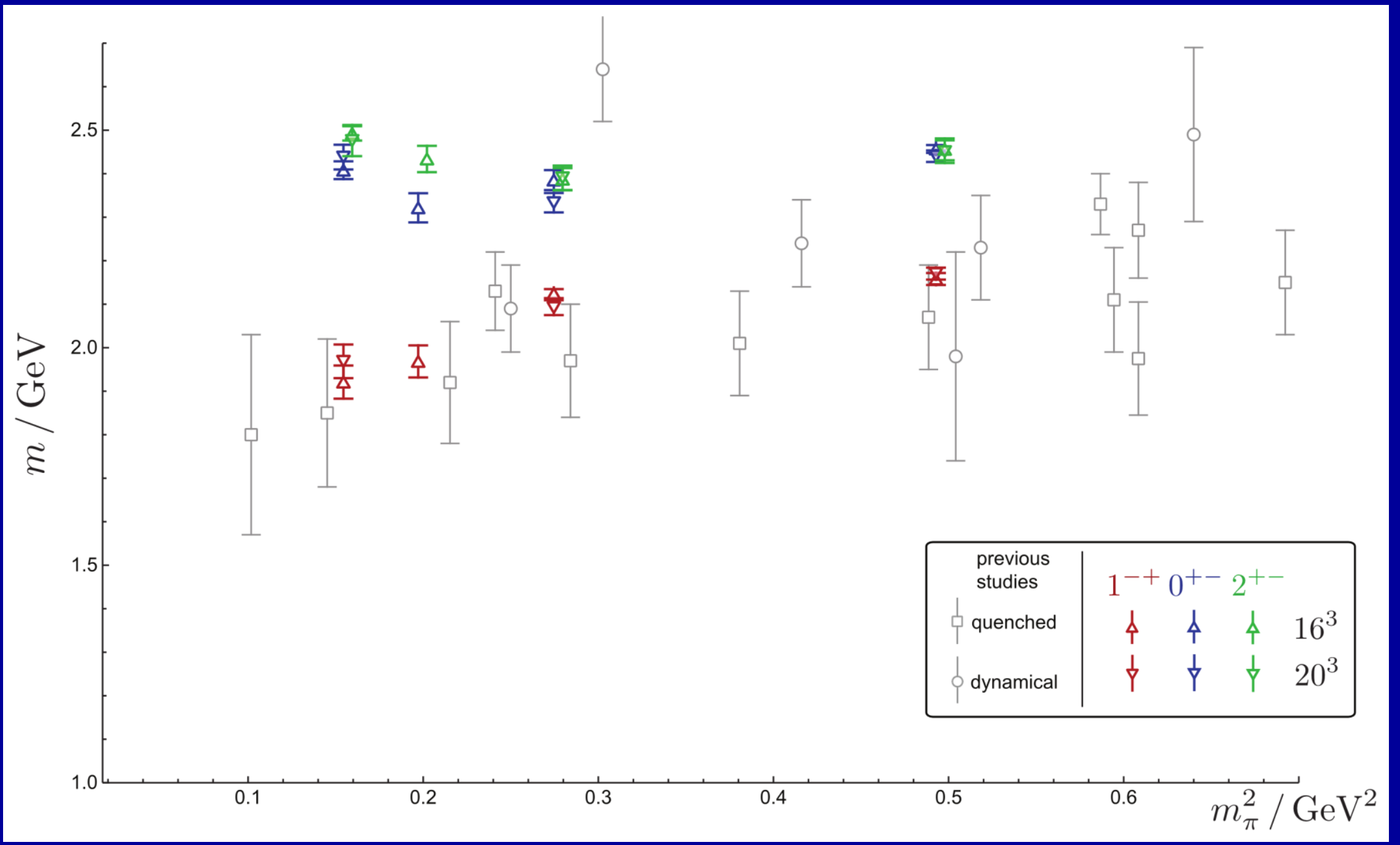




\section{Exotics summary}

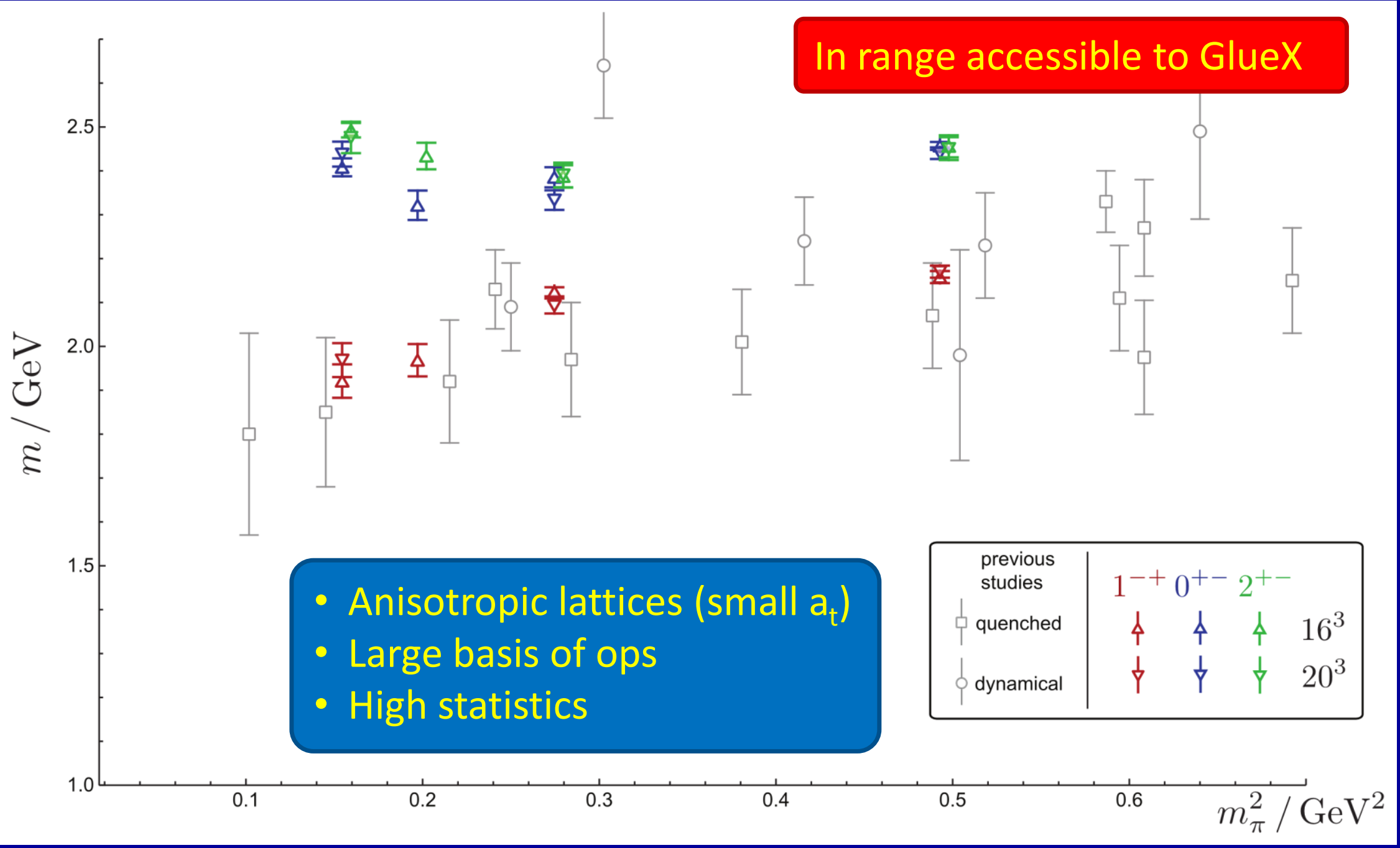




\section{Kaons}

Lower the light quark mass $\left(\mathrm{N}_{\mathrm{f}}=2+1\right)-\mathrm{SU}(3)$ sym breaking

$\begin{array}{llllll}\mathrm{M}_{\pi} / \mathrm{MeV} & 700 & 520 & 440 & 400 & \text { c.f. physical } \\ \mathrm{M}_{\mathrm{K}} / \mathrm{M}_{\pi} & 1 & 1.2 & 1.3 & 1.4 & \mathrm{M}_{\mathrm{K}} / \mathrm{M}_{\pi}=3.5\end{array}$




\section{Kaons}

Lower the light quark mass $\left(\mathrm{N}_{\mathrm{f}}=2+1\right)-\mathrm{SU}(3)$ sym breaking
$\mathrm{M}_{\pi} / \mathrm{MeV} 700$
520
440
400
$\mathrm{M}_{\mathrm{K}} / \mathrm{M}_{\pi} \quad 1$
1.2
1.3
1.4
c.f. physical
$\mathrm{M}_{\mathrm{K}} / \mathrm{M}_{\pi}=3.5$

No longer is C-parity a good quantum number for kaons (or a gen. of C-parity)

Combine $\mathrm{J}^{\mathrm{P}+}$ and $\mathrm{JP}^{\mathrm{P}}$ operators

Physically, axial kaons $\left[K_{1}(1270), K_{1}(1400)\right]$ are a mixture

Suggested mixing angle $\approx 45^{\circ}$ (combination of exp and models)

But... 


\section{Kaons}

Lower th

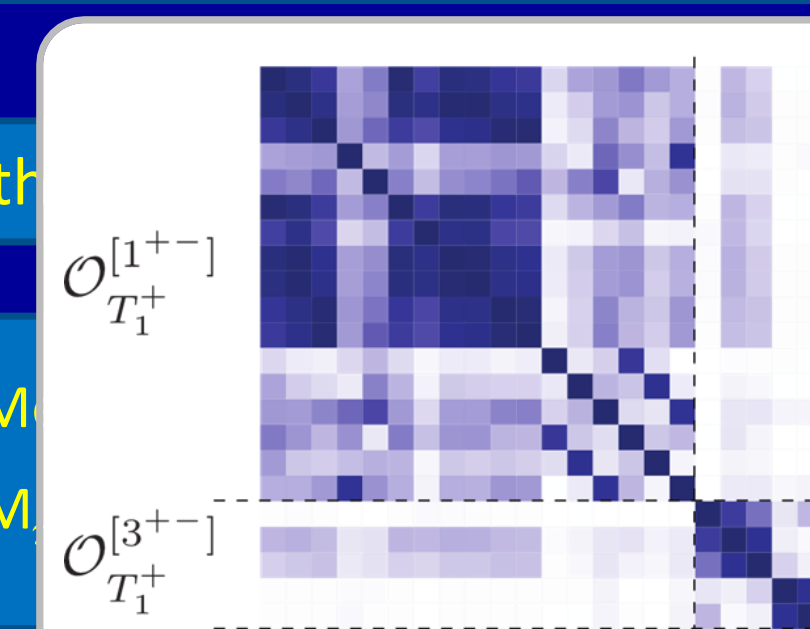

No long

$$
\mathcal{O}_{T_{1}^{+}}^{\left[1^{++}\right]}
$$

Combin

Physica

Suggest $\mathcal{O}_{T_{1}^{+}}^{\left[4^{++}\right.}$

But...

$$
C_{i j} / \sqrt{C_{i i} C_{j j}}
$$

\section{$16^{3}$}

$\mathrm{M}_{\pi} \approx 400 \mathrm{MeV}$

$\mathrm{M}_{\mathrm{K}} / \mathrm{M}_{\pi} \approx 1.4$

c.f. physical

$\mathrm{M}_{\mathrm{K}} / \mathrm{M}_{\pi}=3.5$

pr a gen. of C-parity) 


\section{Kaons - spectrum}

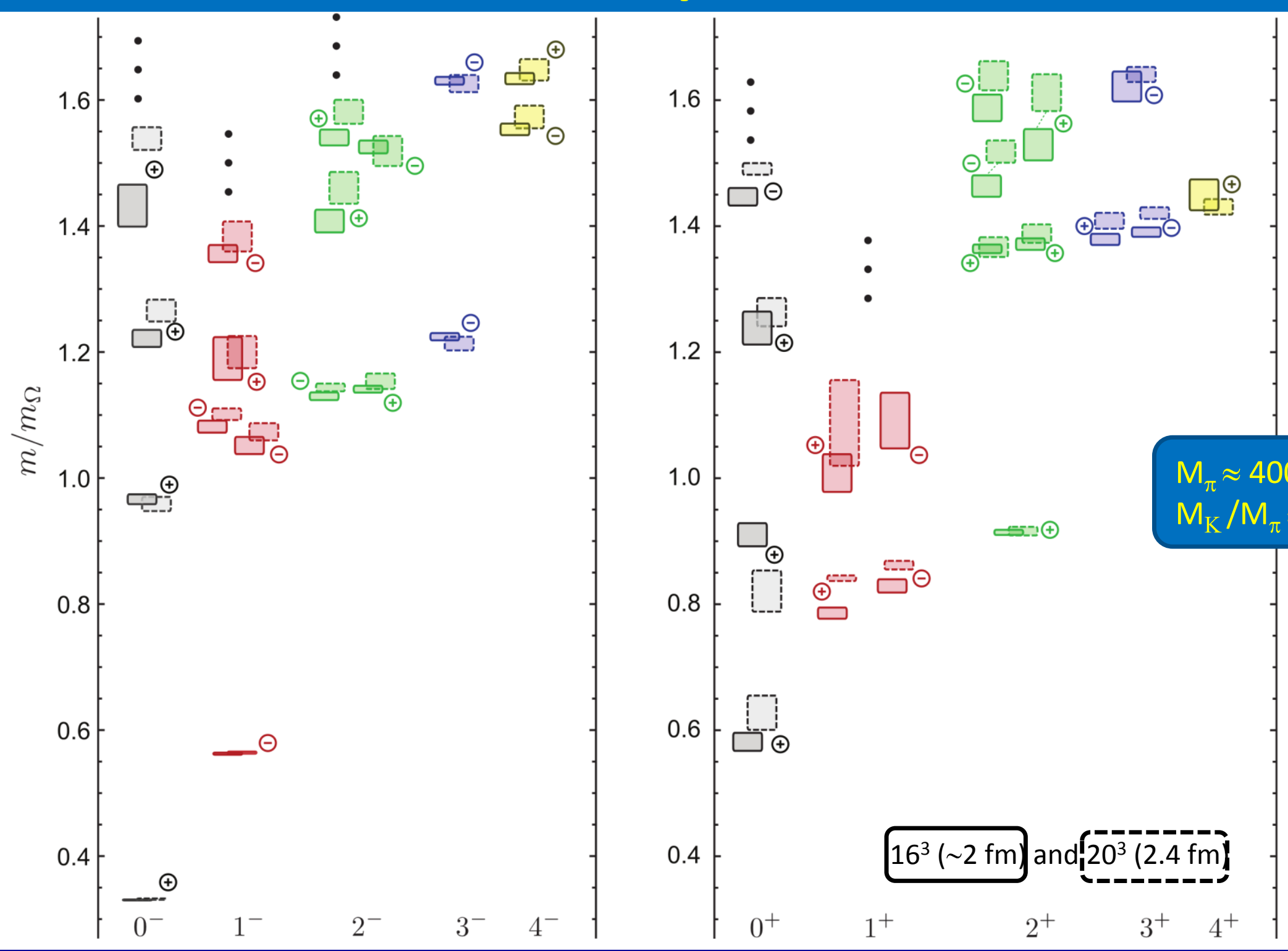




\section{Kaons - Various pion masses}

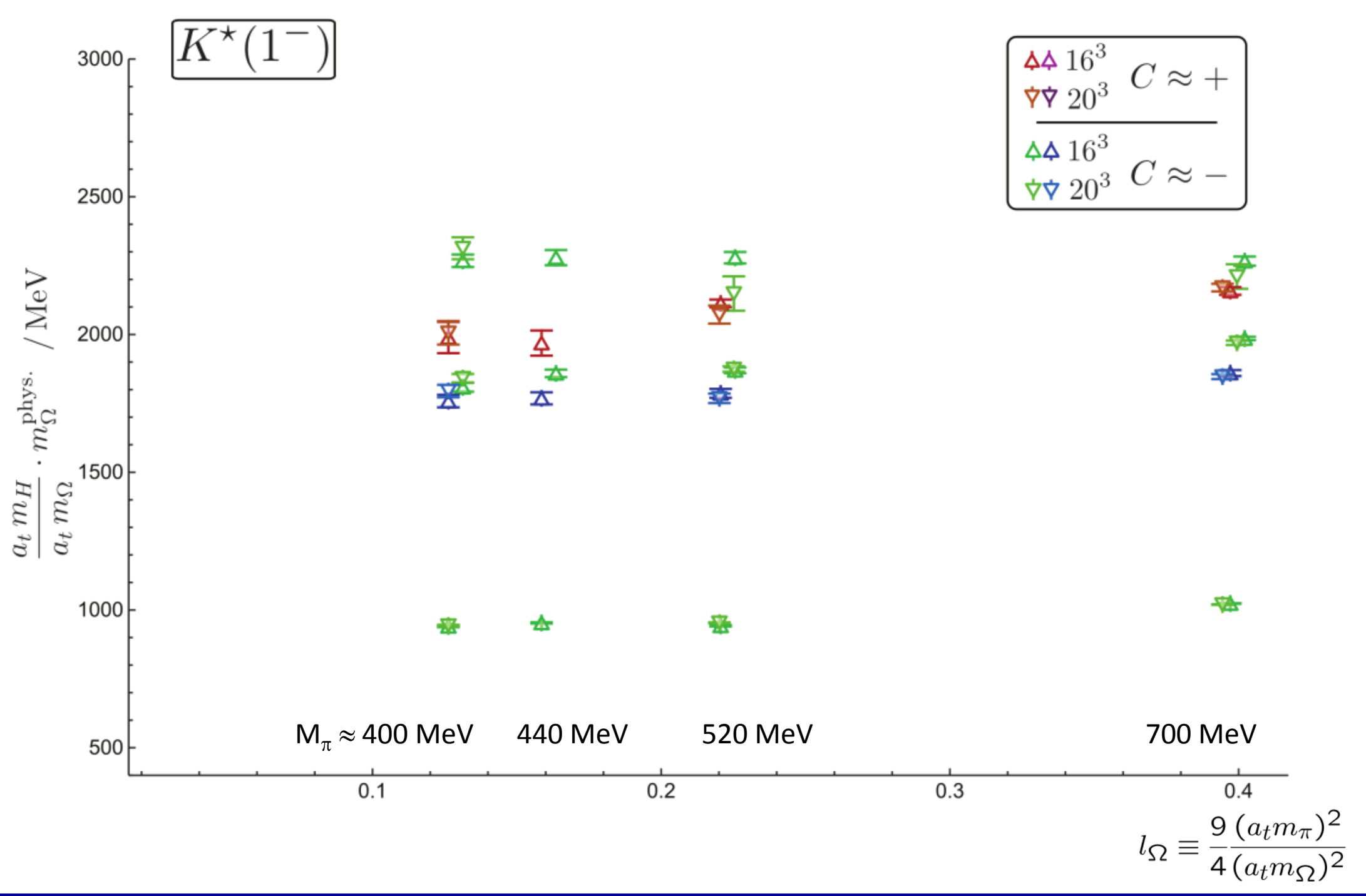




\section{Kaons - Various pion masses}

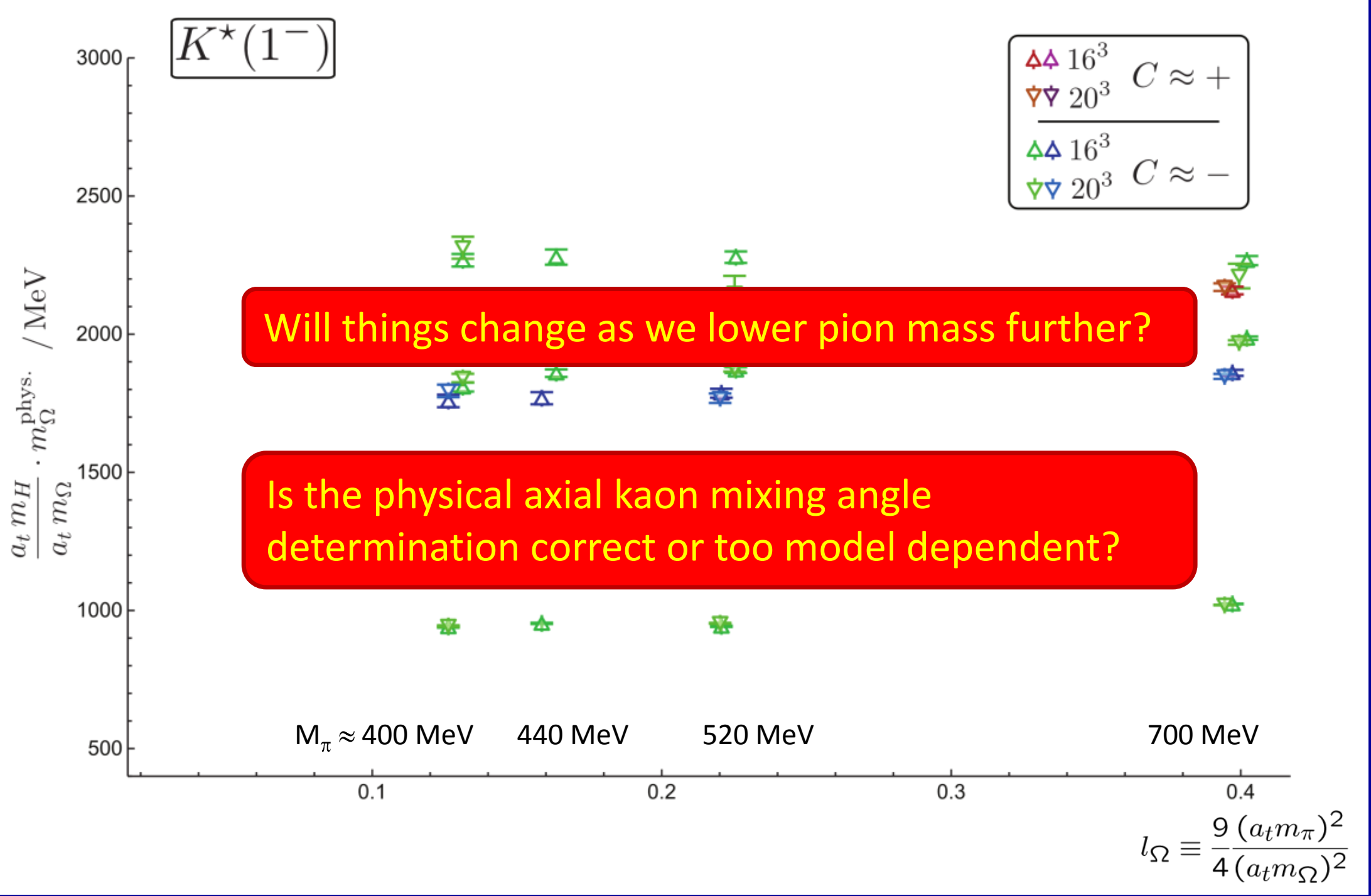




\section{Multi-particle states?}

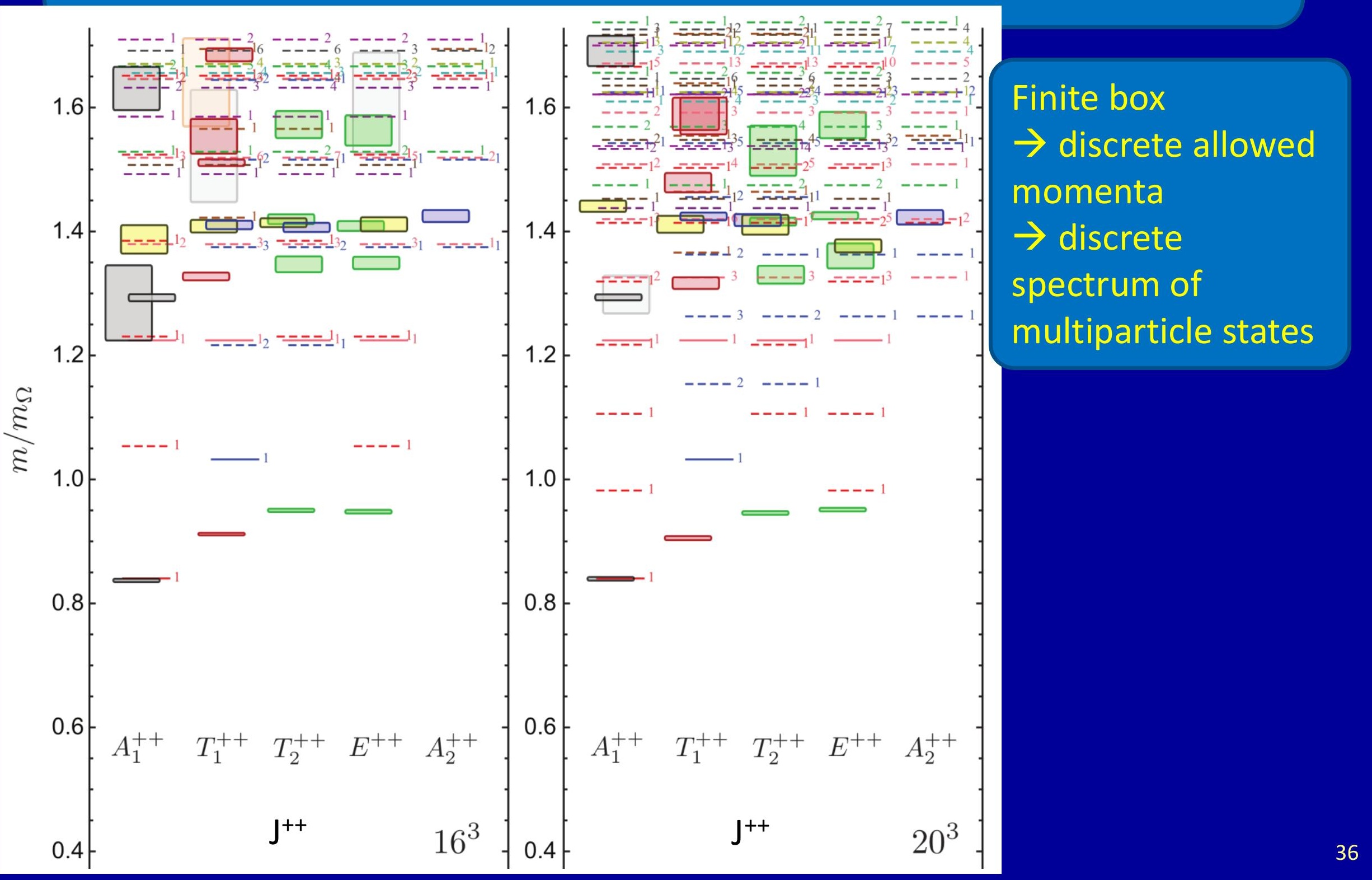




\section{Multi-particle states?}

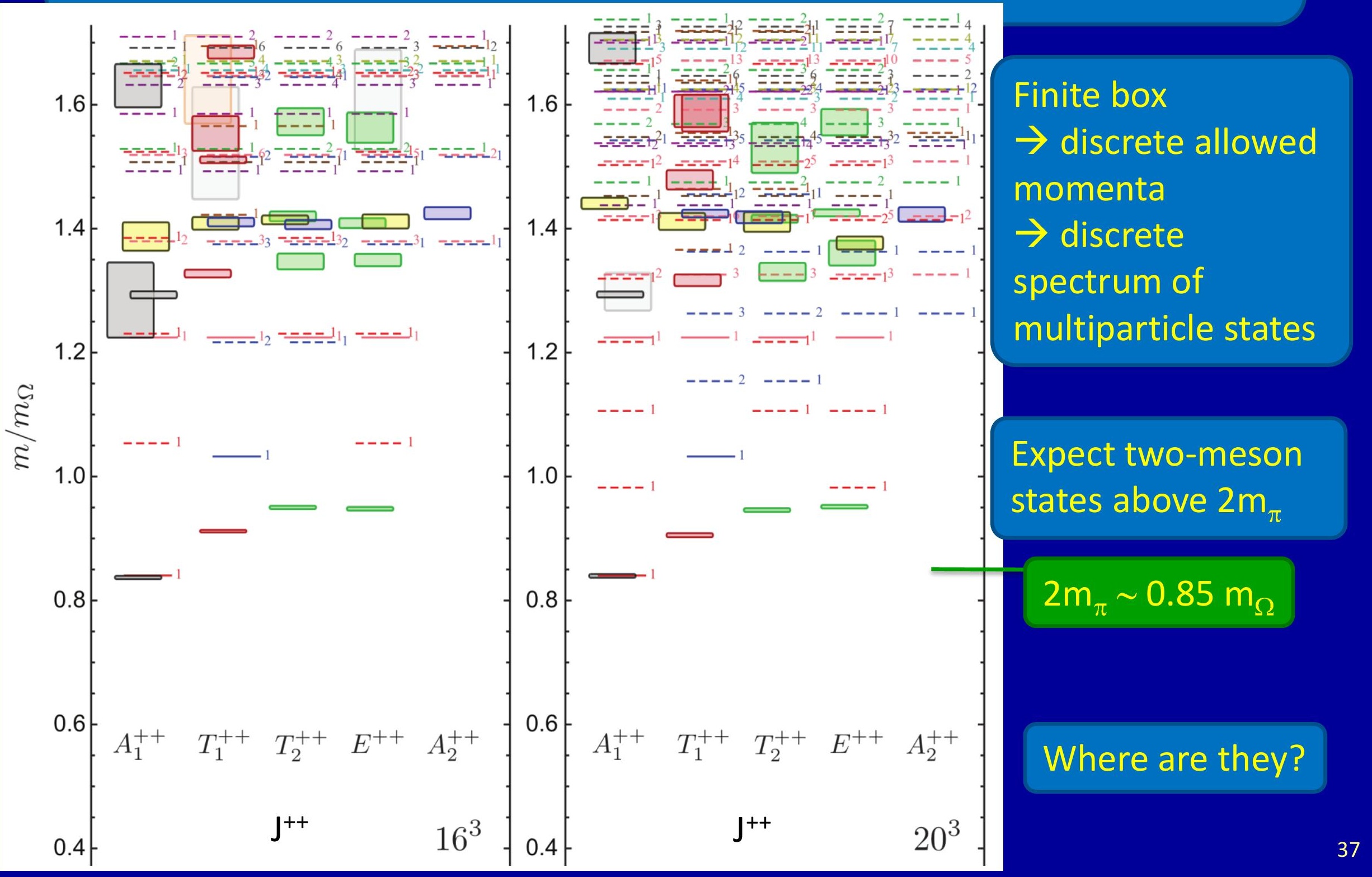




\section{Summary and Outlook}

\section{Summary}

- Our first results on light mesons - technology and method work

- First spin 4 meson extracted and confidently identified on lattice

- Exotics (and non-exotic hybrid candidates)

- Isovectors and kaons 


\section{Summary and Outlook}

\section{Summary}

- Our first results on light mesons - technology and method work

- First spin 4 meson extracted and confidently identified on lattice

- Exotics (and non-exotic hybrid candidates)

- Isovectors and kaons

Outlook - ongoing work

- Multi-meson operators - resonance physics

- Disconnected diagrams - isoscalars and multi-mesons

- Baryons

- Photocouplings

- Lighter pion masses and larger volumes 


\section{Extra Slides}




\section{Kaons - Operator Overlaps (Z's)}

\section{$16^{3}$ \\ $\mathrm{M}_{\pi} \approx 520 \mathrm{MeV}$ \\ $\mathrm{M}_{\mathrm{K}} / \mathrm{M}_{\pi} \approx 1.2$ \\ $16^{3}$ \\ $\mathrm{M}_{\pi} \approx 400 \mathrm{MeV}$ $\mathrm{M}_{\mathrm{K}} / \mathrm{M}_{\pi} \approx 1.4$}

the

(1)

(2)

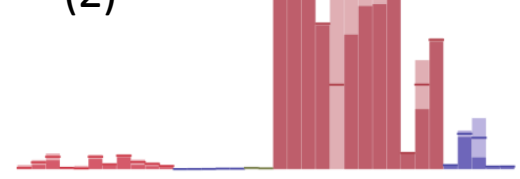
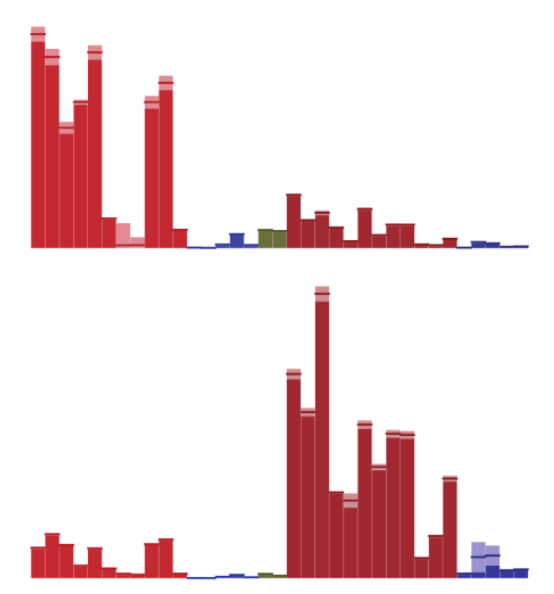

(3)
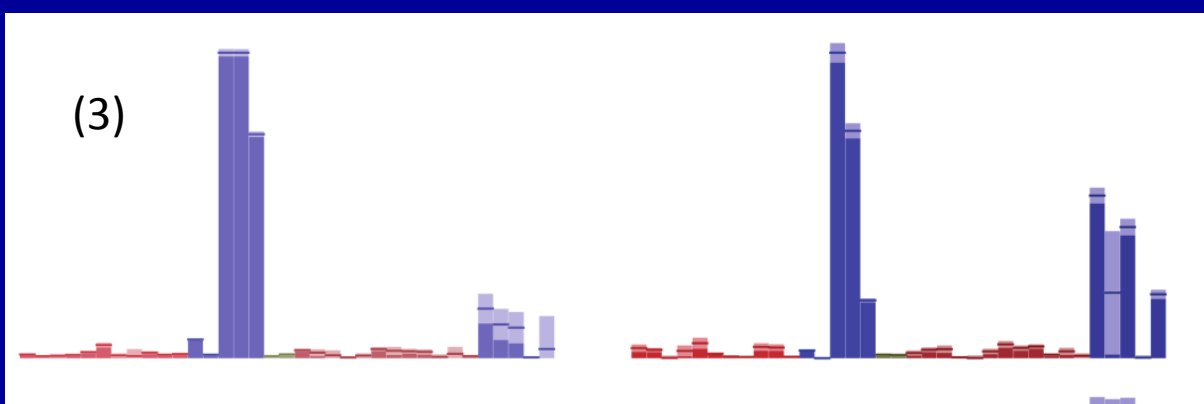

(4)

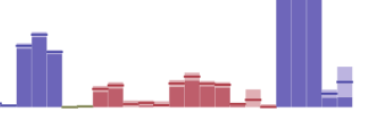

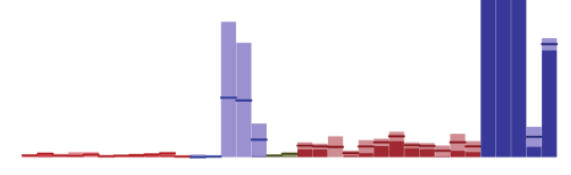

(5)

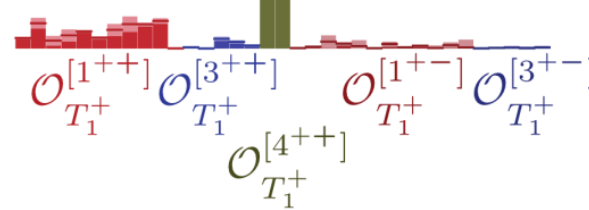




\section{Kaons - Operator Overlaps (Z's)}

\section{$16^{3}$ \\ $\mathrm{M}_{\pi} \approx 520 \mathrm{MeV}$ \\ $\mathrm{M}_{\mathrm{K}} / \mathrm{M}_{\pi} \approx 1.2$

$$
\begin{aligned}
& 16^{3} \\
& \mathrm{M}_{\pi} \approx 400 \mathrm{MeV} \\
& \mathrm{M}_{\mathrm{K}} / \mathrm{M}_{\pi} \approx 1.4
\end{aligned}
$$

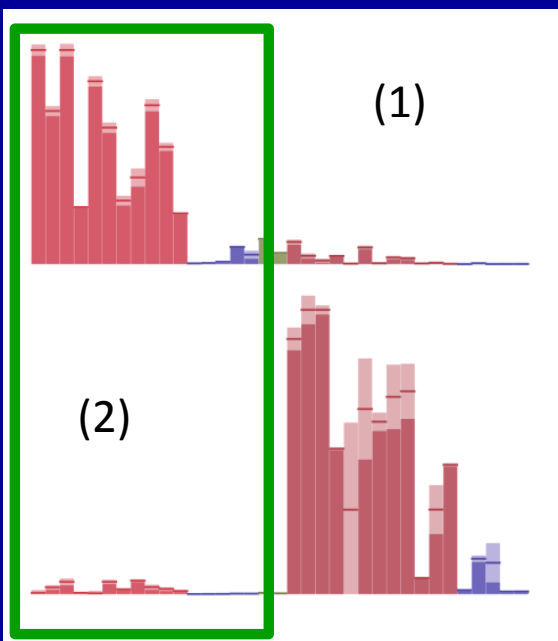

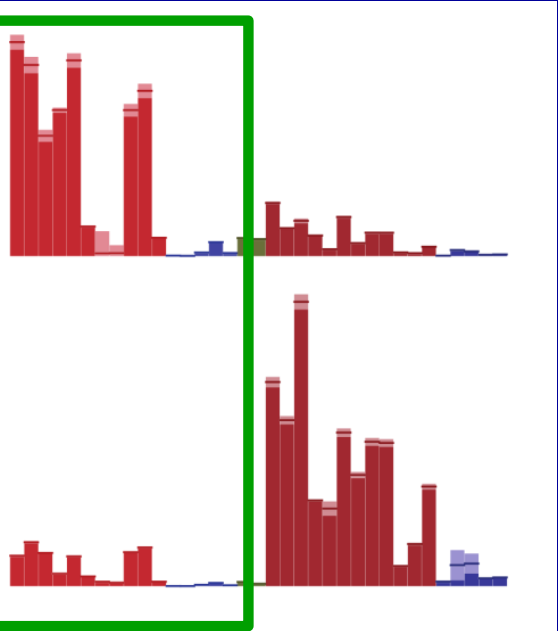

$\mathrm{J}^{++}$
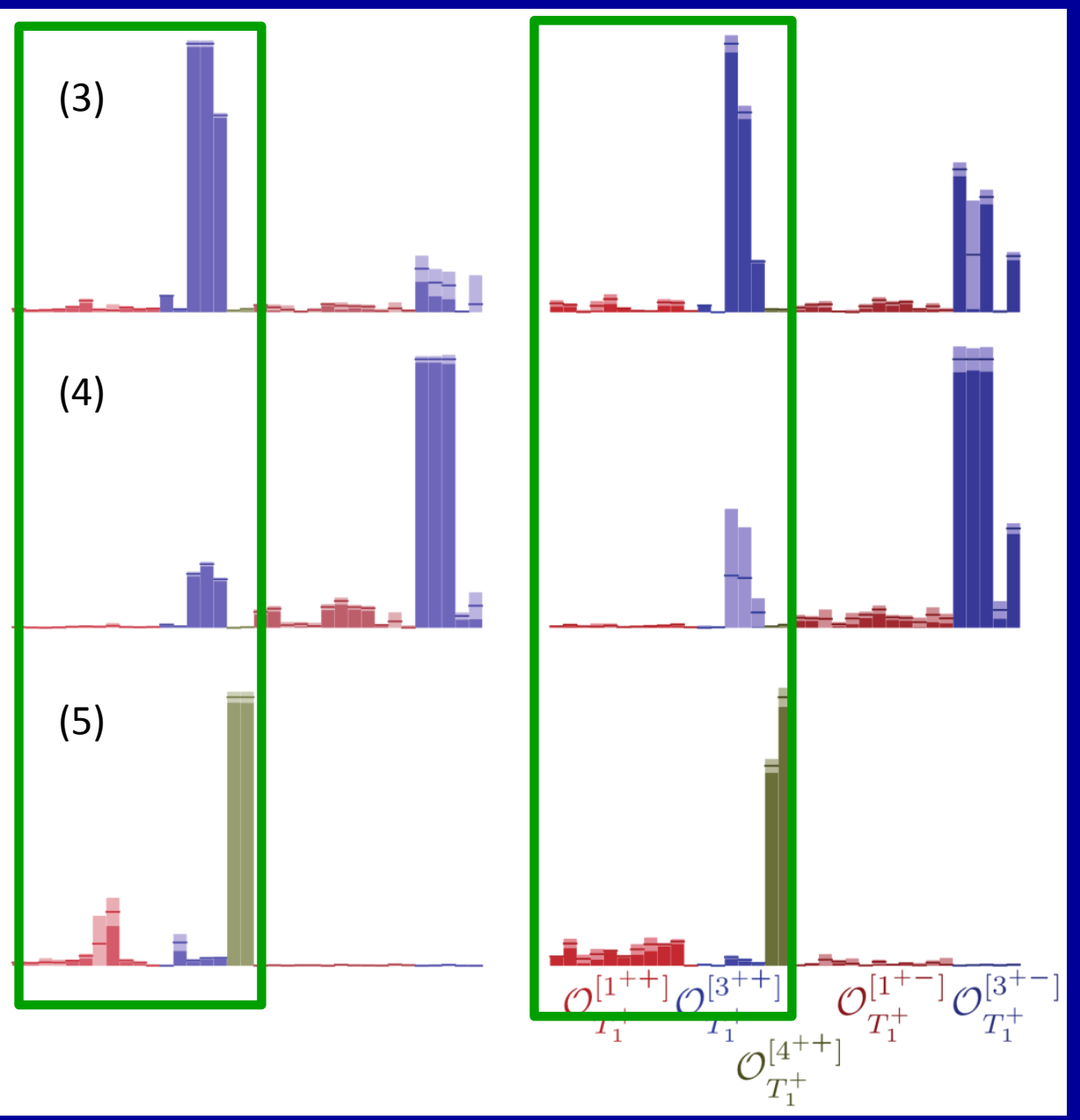


\section{Kaons - Operator Overlaps (Z's)}

$$
\begin{array}{ll}
16^{3} & 16^{3} \\
\mathrm{M}_{\pi} \approx 520 \mathrm{MeV} & \mathrm{M}_{\pi} \approx 400 \mathrm{MeV} \\
\mathrm{M}_{\mathrm{K}} / \mathrm{M}_{\pi} \approx 1.2 & \mathrm{M}_{\mathrm{K}} / \mathrm{M}_{\pi} \approx 1.4
\end{array}
$$
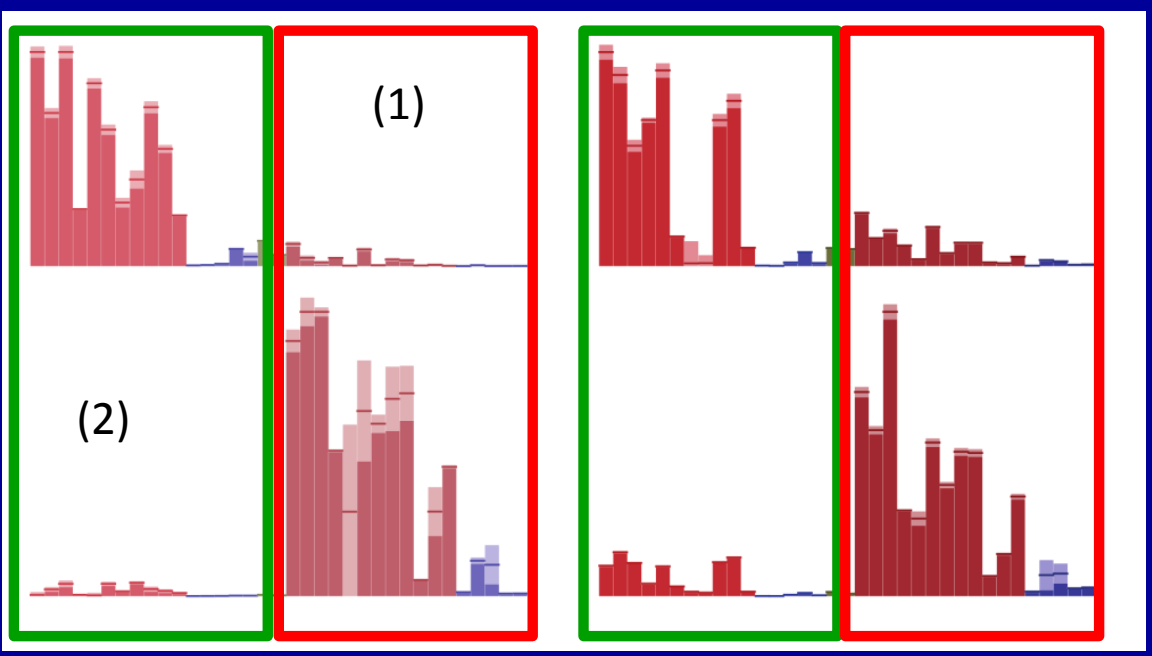

$$
\mathrm{J}^{++}
$$
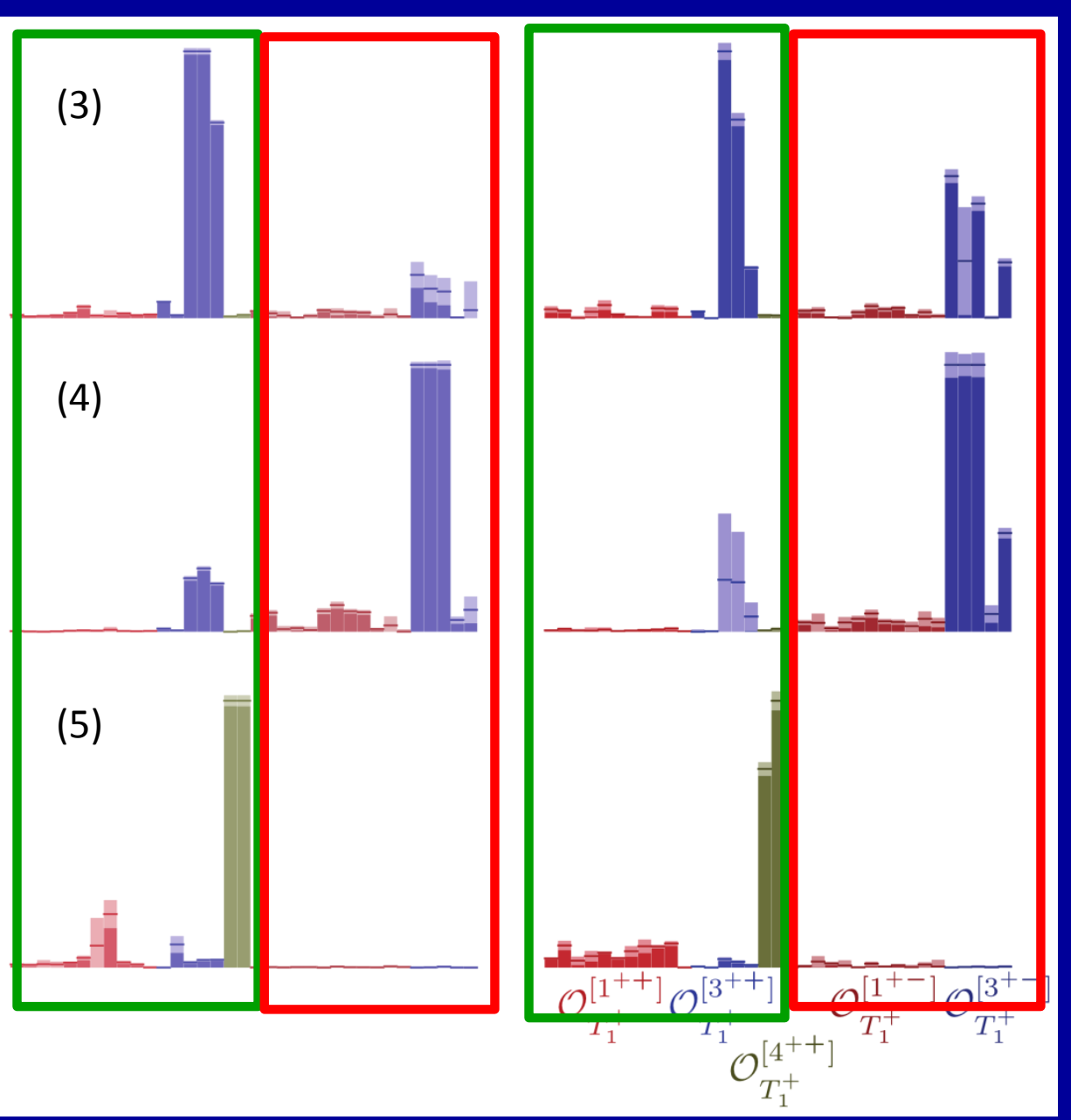


\section{QCD on a Lattice}

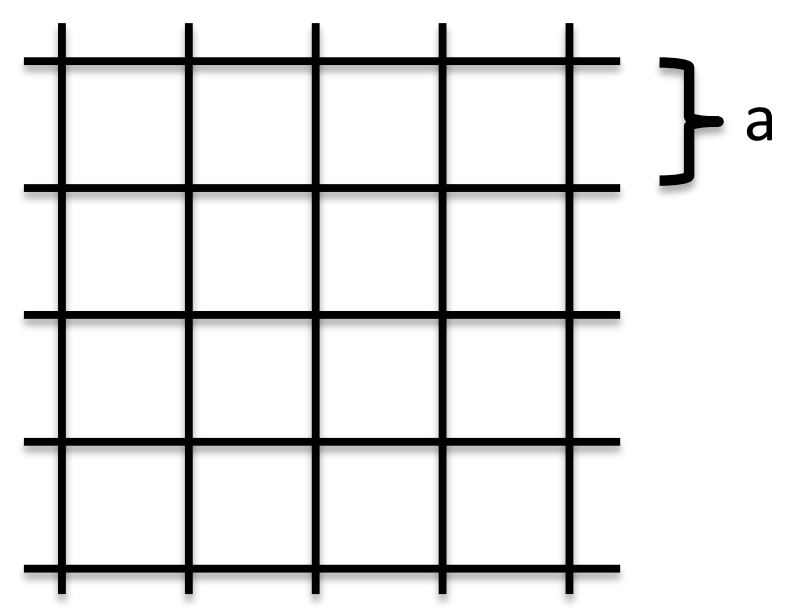

Discretise on a grid (spacing $=\mathrm{a}$ ) - regulator

Finite volume $\rightarrow$ finite no. of d.o.f. 


\section{QCD on a Lattice}

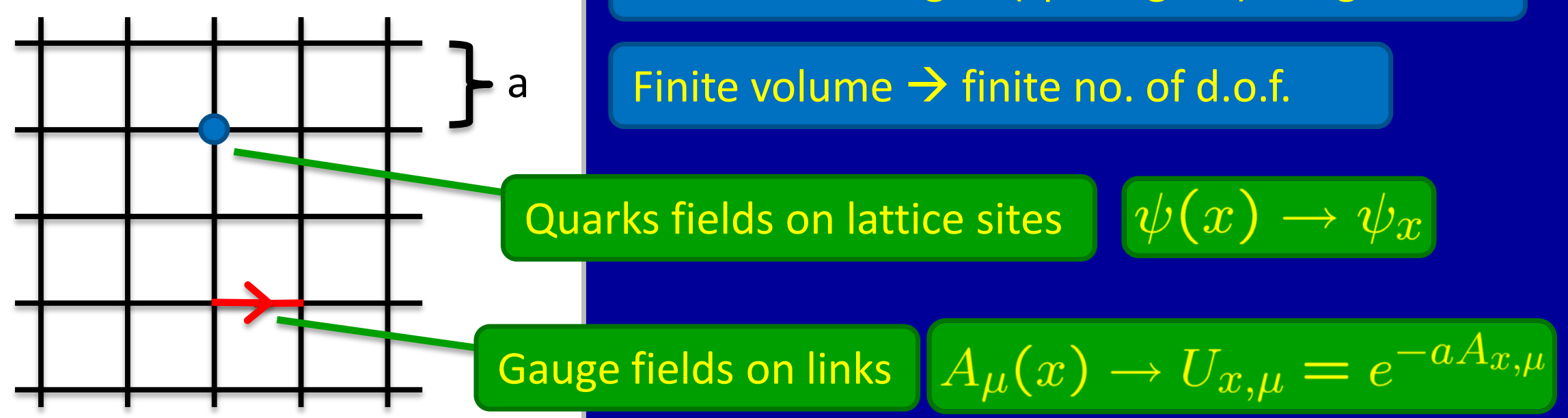




\section{QCD on a Lattice}

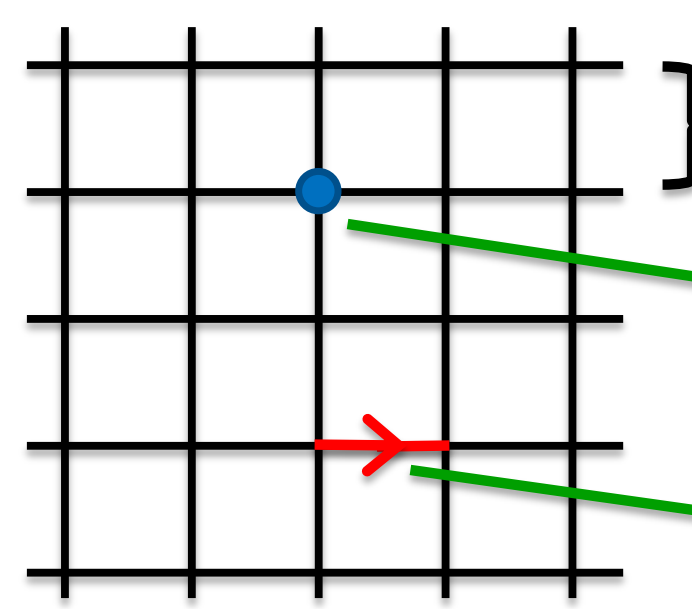

Discretise on a grid (spacing $=$ a) - regulator

Finite volume $\rightarrow$ finite no. of d.o.f.

Quarks fields on lattice sites

$\psi(x) \rightarrow \psi_{x}$

Gauge fields on links

$A_{\mu}(x) \rightarrow U_{x, \mu}=e^{-a A_{x, \mu}}$

Path integral formulation

$\int \mathcal{D} \psi \mathcal{D} \bar{\psi} \mathcal{D} U f(\psi, \bar{\psi}, U) e^{i S[\psi, \bar{\psi}, U]}$ 


\section{QCD on a Lattice}

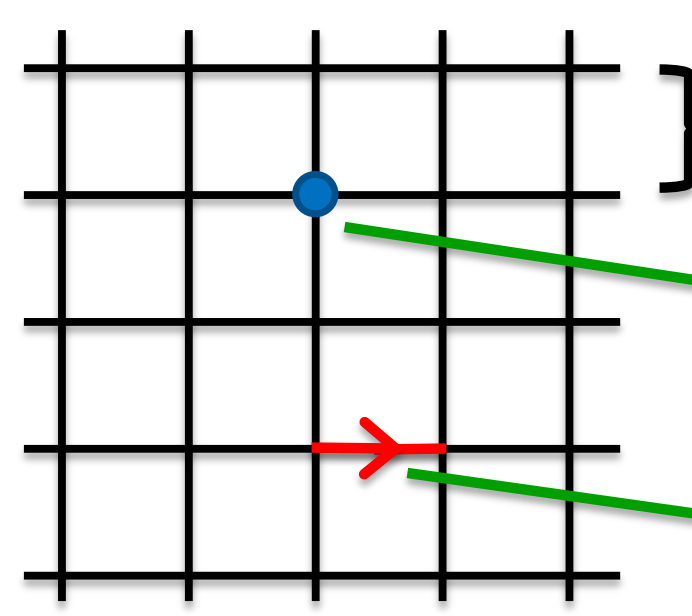

Discretise on a grid (spacing $=$ a) - regulator

Finite volume $\rightarrow$ finite no. of d.o.f.

Quarks fields on lattice sites

$\psi(x) \rightarrow \psi_{x}$

Gauge fields on links $A_{\mu}(x) \rightarrow U_{x, \mu}=e^{-a A_{x, \mu}}$

Path integral formulation

Euclidean time: $\mathrm{t} \rightarrow \mathrm{i} \mathrm{t}$

$\int \mathcal{D} \psi \mathcal{D} \bar{\psi} \mathcal{D} U f(\psi, \bar{\psi}, U) e^{-\widetilde{S}[\psi, \bar{\psi}, U]}$

Do fermion integral analytically, then use importance sampling Monte Carlo 
